\title{
Understanding Color Tuning and Reversible Oxidation of Conjugated Azomethines
}

Sophie Bishop, Marie-Hélène Tremblay, Alexandra Gellé, and W.G. Skene*

Laboratoire de caractérisation photophysique des matériaux conjugués

Département de chimie

Université de Montréal

CP 6128, Centre-ville

Montreal, QC

\section{ELECTRONIC SUPPORTING INFORMATION}




\section{Experimental Details}

All chemicals and solvents were used as received, unless otherwise stated. NMR measurements were done on a $400 \mathrm{MHz}\left({ }^{1} \mathrm{H}\right)$ spectrometer, unless otherwise stated. The absorption measurements were done on a combined UV-vis-NIR spectrometer. Samples were dissolved in anhydrous dichloromethane, unless otherwise stated. Fluorescence measurements were done on a combined steady-state/time-resolved emission spectrometer. Samples were dissolved in dichloromethane and degassed with nitrogen for 20 minutes before each measurement to remove the dissolved oxygen. The samples were then sealed to prevent ambient oxygen diffusion.

Electrochemical measurements were done with a multi-channel potentiostat connected to a three electrodes system using a platinum wire counter-electrode, a silver wire pseudoreference electrode, and a polished platinum working electrode. Ferrocene was used as an internal reference for calibrating the measured potentials against SCE. The redox potential, $\mathrm{E}=1 / 2\left(\mathrm{E}_{\text {forward wave }}+\mathrm{E}_{\text {backward wave }}\right)$, for the reversible $\mathrm{Fc} / \mathrm{Fc}^{+}$couple was taken as +460 mV vs. SCE. ${ }^{1}$ Samples were degassed for 20 minutes with nitrogen before each measurement in order to eliminate the dissolved oxygen. Samples were prepared at a 1 $\mathrm{mM}$ concentration in a $0.10 \mathrm{M}$ solution of tetrabutylammonium hexafluorophosphate $\left(\mathrm{TBAPF}_{6}\right)$ in dichloromethane, unless otherwise described. Additional details and analyses of the compounds can be found elsewhere. ${ }^{2}$ The HOMO and LUMO energy levels were calculated from the onset of the forward electrochemical waves for the oxidation $\left(E_{\mathrm{OX}}{ }^{\text {onset }}\right)$ and reduction $\left(E_{\mathrm{red}}{ }^{\text {onset }}\right)$ processes according to: $\mathrm{HOMO}=-\mathrm{e}\left(E_{\mathrm{ox}}{ }^{\text {onset }}\right.$ 
$(\mathrm{SCE})+4.4), \mathrm{LUMO}=-\mathrm{e}\left(E_{\text {red }}{ }^{\text {onset }}(\mathrm{SCE})+4.4\right) .{ }^{3}$ The electrochemical energy gap $\left(E_{g}{ }^{\mathrm{EC}}\right)$ was calculated as the difference between the two calculated energy levels.

The optimized geometries and corresponding energy levels were theoretically calculated using Gaussian 09 at the DFT level. ${ }^{4}$ The geometries were optimized using the same theoretical level: B3LYP hybrid functional with 6-31G(d) basis set. ${ }^{5-9}$ Optimized geometries were confirmed by frequency calculations with no negative frequencies being calculated. The time-dependent density functional theory (TD-DFT) was used to calculate the optical transitions using the $6-31 \mathrm{G}(\mathrm{d})$ basis set. The first six lowest transitions were calculated.

\section{Synthesis}

2,5-Diaminothiophene-3,4-dicarboxylic acid diethyl ester, ${ }^{10}$ 3,4-dibutylthiophene, ${ }^{11}(E)$ 1,2-di(thiophen-2-yl)ethene, ${ }^{12} \quad$ 3,4-dibutylthiophene-2-carbaldehyde, ${ }^{11}$ dibutylthiophene-2,5-dicarbaldehyde, ${ }^{11} \quad$ 2,5-dibromo-3,4-dibutylthiophene, ${ }^{13} \quad\left[2,2^{\prime}-\right.$ bithiophene]-5,5'-dicarbaldehyde, ${ }^{14}$ [2,2':5',2"-terthiophene]-5,5"-dicarbaldehyde), ${ }^{15}(E)$ 5,5'-(ethene-1,2-diyl)bis(thiophene-2-carbaldehyde), ${ }^{12}$ and (5-(diethyl-5-amino-thiophen3,4-dicarboxylate-2-ylimino)-methyl)((thiophen-2-yl)methylene)-diethylthiophene-3,4-

dicarboxylate-2,5-diamine ${ }^{16}$ were prepared according to known methods. 3,4Dibromothiophene, bithiophene, terthiophene and 2,5-thiophenedicarboxaldehyde were commercially available and they were used without further purification. 
carbaldehyde (70 mg, $3.1 \mathrm{mmol})$ was transferred to a $25 \mathrm{~mL}$ round bottom flask equipped with a stir bar using a 1:1 (v/v) ethyl acetate/chloroform mixture (7 mL). Nbromosuccinimide $(60 \mathrm{mg}, 3.4 \mathrm{mmol})$ was added and the mixture was stirred at room temperature for $16 \mathrm{~h}$ under a $\mathrm{N}_{2}$ atmosphere. The contents of the flask were then poured into cold distilled water $(25 \mathrm{~mL})$, transferred into a separatory funnel, and extracted with dichloromethane $(3 \times 20 \mathrm{~mL})$. The organic fraction was washed with distilled water $(1 \mathrm{x}$ $20 \mathrm{~mL})$, a saturated aqueous $\mathrm{NaHCO}_{3}$ solution $(1 \times 25 \mathrm{~mL})$, and a saturated aqueous $\mathrm{NaCl}$ solution $\left(1 \times 25 \mathrm{~mL}\right.$ ). It was then dried with anhydrous $\mathrm{MgSO}_{4}$. The solid was removed by filtering and the filtrate was evaporated in vacuo. The resulting oil was purified by silica gel column chromatography $(74 \mathrm{mg}, 79 \%) .{ }^{1} \mathrm{H}-\mathrm{NMR}$ (acetone- $\left.d_{6}\right): \delta=$ $10.02(\mathrm{~s}, 1 \mathrm{H}), 3.02(\mathrm{t}, 2 \mathrm{H}, J=8.0 \mathrm{~Hz}), 2.63(\mathrm{t}, 2 \mathrm{H}, J=7.8 \mathrm{~Hz}), 1.65-1.41(\mathrm{~m}, 8 \mathrm{H}), 0.96$ $(\mathrm{dt}, 6 \mathrm{H}, J=7.2 \mathrm{~Hz}, 2.4 \mathrm{~Hz}) .{ }^{13} \mathrm{C}-\mathrm{NMR}\left(\mathrm{CDCl}_{3}\right): \delta=180.9,151.0,143.5,138.2,122.1$, 34.2, 27.4, 27.2, 13.6, 13.5. HRMS(+) calculated for $\left[\mathrm{C}_{13} \mathrm{H}_{19} \mathrm{BrOS}\right] \mathrm{H}^{+}$: 303.0413 , found: 303.0409 .

\section{3,3',4,4'-Tetrabutyl-[2,2'-bithiophene]-5,5'-dicarboxaldehyde.}

dibutylthiophene-2-carboxaldehyde $(0.74 \mathrm{~g}, 2.4 \mathrm{mmol})$ was transferred into a $50 \mathrm{~mL}$ double-necked round bottom flask equipped with a magnetic stirrer and a condensing tube using anhydrous toluene (3 mL). Diisopropylethylamine (40 $\mu \mathrm{L}, 0.42 \mathrm{mmol})$, tetrabutylammonium bromide $(37 \mathrm{mg}, 0.11 \mathrm{mmol})$, and palladium (II) acetate $(1.3 \mathrm{mg}$, $0.006 \mathrm{mmol})$ were added. The mixture was stirred and three vacuum- $\mathrm{N}_{2}$ cycles were completed before refluxing in an oil bath at $105^{\circ} \mathrm{C}$ for $4 \mathrm{~h}$. The reaction mixture was 
then heated at $77{ }^{\circ} \mathrm{C}$ for $15.5 \mathrm{~h}$, followed by heating at $105{ }^{\circ} \mathrm{C}$ for $3.5 \mathrm{~h}$. Afterwards, the contents of the flask were poured into cold distilled water $(10 \mathrm{~mL})$ before being transferred to a separatory funnel, and extracted with dichloromethane $(3 \times 10 \mathrm{~mL})$. The organic fraction was washed with distilled water $(10 \mathrm{~mL})$ and a saturated aqueous $\mathrm{NaCl}$ solution $(2 \times 10 \mathrm{~mL})$. It was then dried with anhydrous $\mathrm{MgSO}_{4}$, filtered, and the solvent was removed in vacuo. The resulting oil was purified by silica gel column chromatography $(6.8 \mathrm{mg}, 1.2 \%) .{ }^{1} \mathrm{H}-\mathrm{NMR}$ (acetone- $\left.d_{6}\right): \delta=10.15(\mathrm{~s}, 2 \mathrm{H}), 3.06(\mathrm{t}, 4 \mathrm{H}, J$ $=8.0 \mathrm{~Hz}), 2.59(\mathrm{t}, 4 \mathrm{H}, J=4.0 \mathrm{~Hz}), 1.71-1.63(\mathrm{~m}, 4 \mathrm{H}), 1.51-1.41(\mathrm{~m}, 8 \mathrm{H}), 1.33-1.23$ $(\mathrm{m}, 4 \mathrm{H}), 0.97(\mathrm{t}, 6 \mathrm{H}, J=7.8 \mathrm{~Hz}), 0.82(\mathrm{t}, 6 \mathrm{H}, J=7.6 \mathrm{~Hz}) .{ }^{13} \mathrm{C}-\mathrm{NMR}\left(\mathrm{CDCl}_{3}\right): \delta=182.3$, $151.9,144.2,138.4,138.2,34.6,32.8,27.2,26.9,22.8,22.7,13.8,13.6 . \operatorname{HRMS}(+)$ calculated for $\left[\mathrm{C}_{26} \mathrm{H}_{38} \mathrm{O}_{2} \mathrm{~S}_{2}\right] \mathrm{H}^{+}$: 447.2386, found: 447.2396.

\section{3,3',3',4,4',4'"-Hexabutyl-[2,2':5',2'-terthiophene]-5,5'"-dicarboxaldehyde.}

Phosphoryl chloride $(0.31 \mathrm{~mL}, 3.4 \mathrm{mmol})$ was combined with dichloroethane $(10 \mathrm{~mL})$ in a $25 \mathrm{~mL}$ Schlenk tube equipped with a stir bar. The solution was then cooled to $0{ }^{\circ} \mathrm{C}$ in an ice bath and anhydrous $N, N$-dimethylformamide $(0.26 \mathrm{~mL}, 3.4 \mathrm{mmol})$ was added. The solution was then warmed to room temperature. After 45 min., 3,3',3",4,4',4"-hexabutyl2,2':5',2"-terthiophene $(0.25 \mathrm{~g}, 0.43 \mathrm{mmol})$ was added, followed by heating in an oil bath at $65{ }^{\circ} \mathrm{C}$ for $15 \mathrm{~h}$. The mixture was poured into distilled water, transferred into a separatory funnel and extracted with dichloromethane $(3 \times 25 \mathrm{~mL})$. The organic fraction was washed with distilled water (1 x $50 \mathrm{~mL}$ ), dried with anhydrous $\mathrm{MgSO}_{4}$, filtered, and the solvent was then removed in vacuo. The resulting orange oil was purified by silica gel column chromatography $(0.22 \mathrm{~g}, 80 \%) .{ }^{1} \mathrm{H}-\mathrm{NMR}$ (acetone- $\left.d_{6}\right): \delta=10.14(\mathrm{~s}, 2 \mathrm{H}), 3.05(\mathrm{t}$, 
$4 \mathrm{H}, J=7.6 \mathrm{~Hz}), 2.62(\mathrm{q}, 8 \mathrm{H}, J=8.0 \mathrm{~Hz}), 1.71-1.64(\mathrm{~m}, 4 \mathrm{H}), 1.53-1.43(\mathrm{~m}, 12 \mathrm{H}), 1.36-$ $1.24(\mathrm{~m}, 8 \mathrm{H}), 0.97(\mathrm{t}, 6 \mathrm{H}, J=7.2 \mathrm{~Hz}), 0.84(\mathrm{dt}, 12 \mathrm{H}, J=7.4 \mathrm{~Hz}, 3.0 \mathrm{~Hz}) .{ }^{13} \mathrm{C} \mathrm{NMR}$ (acetone- $\left.d_{6}\right): \delta=184.2,153.6,145.8,144.6,140.2,140.0,131.3,36.4,34.6,34.5,29.3$, 28.6, 24.4, 24.3, 15.1, 15.02, 15.01. $\mathrm{HRMS}(+)$ calculated for $\left[\mathrm{C}_{38} \mathrm{H}_{56} \mathrm{O}_{2} \mathrm{~S}_{3}\right] \mathrm{H}^{+}: 641.3515$, found 641.3504 .

\section{Representative procedure for the synthesis of $1 \mathrm{~B}-4$.}

Tetraethyl-5,5'-((1E,1'E)-((3,4-dibutylthiophene-2,5-diyl)bis(methanylylidene))bis(azanylylidene))bis(2-aminothiophene-3,4-dicarboxylate) (1B). 3,4-Dibutylthiophene2,5-dicarbaldehyde (0.11 g, $0.45 \mathrm{mmol})$ and 2,5-diaminothiophene-3,4-dicarboxylic acid diethyl ester $(0.45 \mathrm{~g}, 1.7 \mathrm{mmol})$ were combined in a test tube equipped with a stir bar. The reagents were dissolved in anhydrous ethanol $(2 \mathrm{~mL})$ followed by the addition of a catalytic amount of trifluoroacetic acid. The mixture was then stirred for $2.5 \mathrm{~h}$. The solvent was removed in vacuo and the resulting solid was dissolved in a minimal amount of acetone $(1 \mathrm{~mL})$ before precipitating in cold hexane $(25 \mathrm{~mL})$. The precipitate was filtered and washed with cold hexane $(3 \times 5 \mathrm{~mL})$ before purifying by silica gel column chromatography that was pre-rinsed with $1 \%$ triethylamine $(0.27 \mathrm{~g}, 81 \%) .{ }^{1} \mathrm{H}-\mathrm{NMR}$ (acetone- $\left.d_{6}\right): \delta=8.17(\mathrm{~s}, 2 \mathrm{H}), 7.51(\mathrm{br}, 2 \mathrm{H}), 4.35(\mathrm{q}, 4 \mathrm{H}, J=7.2 \mathrm{~Hz}), 4.21(\mathrm{q}, 4 \mathrm{H}, J=7.2$ $\mathrm{Hz}), 1.60-1.39(\mathrm{~m}, 14 \mathrm{H}), 1.27(\mathrm{t}, 10 \mathrm{H}, J=7.2 \mathrm{~Hz}), 0.97(\mathrm{t}, 6 \mathrm{H}, J=7.2 \mathrm{~Hz}) .{ }^{13} \mathrm{C}-\mathrm{NMR}$ $\left(\mathrm{CDCl}_{3}\right): \delta=165.2,164.5,159.5,146.5,144.5,140.1,134.6,129.7,103.4,61.6,60.3$ 33.9, 26.5, 22.6, 14.5, 14.2, 13.9. $\mathrm{HRMS}(+)$ calculated for $\left[\mathrm{C}_{34} \mathrm{H}_{44} \mathrm{~N}_{4} \mathrm{O}_{8} \mathrm{~S}_{3}\right] \mathrm{H}^{+}:$733.2394, found 733.2370 . 
Tetraethyl-5,5'-((1E,1'E)-([2,2'-bithiophene]-5,5'-diylbis(methanylylidene))bis(azanylylidene))bis(2-aminothiophene-3,4-dicarboxylate) (2A). ${ }^{1} \mathrm{H}-\mathrm{NMR}\left(\mathrm{DMSO}-d_{6}\right): \delta=$ $8.21(\mathrm{~s}, 2 \mathrm{H}), 7.95(\mathrm{br}, 4 \mathrm{H}), 7.54(\mathrm{~d}, 2 \mathrm{H}, J=3.6 \mathrm{~Hz}), 7.48(\mathrm{~d}, 2 \mathrm{H}, J=3.6 \mathrm{~Hz}), 4.28(\mathrm{q}, 4 \mathrm{H}$, $J=7.2 \mathrm{~Hz}), 4.14(\mathrm{q}, 4 \mathrm{H}, J=7.2 \mathrm{~Hz}), 1.33(\mathrm{t}, 6 \mathrm{H}, J=7.0 \mathrm{~Hz}), 1.20(\mathrm{t}, 6 \mathrm{H}, J=7.2 \mathrm{~Hz})$.

${ }^{13}$ C-NMR (DMSO- $\left.d_{6}\right): \delta=164.6,163.1,161.0,145.3,141.9,139.3,133.4,131.5,129.7$, 126.2, 100.1, 60.7, 59.5, 14.1, 14.0. $\operatorname{HRMS(+)~calculated~for~}\left[\mathrm{C}_{30} \mathrm{H}_{30} \mathrm{~N}_{4} \mathrm{O}_{8} \mathrm{~S}_{4}\right] \mathrm{H}^{+}$: 703.1019, found 703.1009.

Tetraethyl-5,5'-((1E,1'E)-((3,3',4,4'-tetrabutyl-[2,2'-bithiophene]-5,5'-diyl)bis(methanylylidene))bis(azanylylidene))bis(2-aminothiophene-3,4-dicarboxylate) (2B). ${ }^{1} \mathrm{H}$ NMR $\left(\mathrm{CDCl}_{3}\right): \delta=8.08(\mathrm{~s}, 2 \mathrm{H}), 6.24(\mathrm{br}, 4 \mathrm{H}), 4.39-4.32(\mathrm{~m}, 4 \mathrm{H}), 4.22(\mathrm{q}, 4 \mathrm{H}, J=7.0$ Hz), 2.74-2.69 (m, 4H), 2.51-2.42 (m, 4H), 1.41-1.36 (m, 14H), 1.30-1.23 (m, 14H), $0.95(\mathrm{t}, 6 \mathrm{H}, J=7.2 \mathrm{~Hz}), 0.84-0.79(\mathrm{~m}, 6 \mathrm{H})$. HRMS $(+)$ calculated for $\left[\mathrm{C}_{46} \mathrm{H}_{62} \mathrm{~N}_{4} \mathrm{O}_{8} \mathrm{~S}_{4}\right] \mathrm{H}^{+}$: 927.3523, found 927.3530.

Tetraethyl-5,5'-((1E,1'E)-([2,2':5',2' '-terthiophene]-5,5''-diylbis(methanylylidene))bis(azanylylidene))bis(2-aminothiophene-3,4-dicarboxylate) (3A). ${ }^{1} \mathrm{H}-\mathrm{NMR}$ (DMSO$\left.d_{6}\right): \delta=8.21(\mathrm{~s}, 2 \mathrm{H}), 7.93(\mathrm{br}, 4 \mathrm{H}), 7.54(\mathrm{~d}, 2 \mathrm{H}, J=4.2 \mathrm{~Hz}), 7.45(\mathrm{~s}, 2 \mathrm{H}), 7.43(\mathrm{~d}, 2 \mathrm{H}, J=$ $4.2 \mathrm{~Hz}), 4.28(\mathrm{q}, 4 \mathrm{H}, J=7.0 \mathrm{~Hz}), 4.14(\mathrm{q}, 4 \mathrm{H}, J=7.0 \mathrm{~Hz}), 1.33(\mathrm{t}, 6 \mathrm{H}, J=7.0 \mathrm{~Hz}), 1.20$ $(\mathrm{t}, 6 \mathrm{H}, J=7.0 \mathrm{~Hz}) .{ }^{13} \mathrm{C}-\mathrm{NMR}\left(\mathrm{DMSO}-d_{6}\right): \delta=164.6,163.2,161.0,145.5,141.3,139.2$, $136.0,133.5,131.7,129.5,126.7,125.6,100.1,60.8,59.5,14.2,14.1 . \operatorname{HRMS}(+)$ calculated for $\left[\mathrm{C}_{34} \mathrm{H}_{32} \mathrm{~N}_{4} \mathrm{O}_{8} \mathrm{~S}_{5}\right] \mathrm{H}^{+}$: 785.0896, found 785.0898. 
Tetraethyl-5,5'-((1E,1'E)-((3,3',3',4,4',4'-hexabutyl-[2,2':5',2'-terthiophene]-5,5"'diyl)bis(methanylylidene))bis(azanylylidene))bis(2-aminothiophene-3,4-dicarboxylate) (3B). ${ }^{1} \mathrm{H}-\mathrm{NMR}\left(\mathrm{CDCl}_{3}\right): \delta=8.12(\mathrm{~s}, 2 \mathrm{H}), 6.27(\mathrm{~s}, 4 \mathrm{H}), 4.38(\mathrm{q}, 4 \mathrm{H}, J=7.2 \mathrm{~Hz})$, $4.24(\mathrm{q}, 4 \mathrm{H}, J=7.2 \mathrm{~Hz}), 2.74(\mathrm{t}, 4 \mathrm{H}, J=8.0 \mathrm{~Hz}), 2.53-2.46(\mathrm{~m}, 8 \mathrm{H}), 1.57-1.51(\mathrm{~m}, 8 \mathrm{H})$, $1.46-1.36(\mathrm{~m}, 18 \mathrm{H}), 1.30(\mathrm{t}, 10 \mathrm{H}, J=7.2 \mathrm{~Hz}), 0.97(\mathrm{t}, 6 \mathrm{H}, J=7.2 \mathrm{~Hz}), 0.84(\mathrm{dt}, 12 \mathrm{H}, J=$ $7.2 \mathrm{~Hz}, 4.0 \mathrm{~Hz}) .{ }^{13} \mathrm{C}-\mathrm{NMR}\left(\mathrm{CDCl}_{3}\right): \delta=165.3,164.5,159.0,146.3,145.2,142.8,142.1$, $136.5,135.0,134.7,129.8,128.4,103.2,61.5,60.2,34.2,32.9,32.8,29.9,27.7,27.2$, 27.1, 22.8, 22.7, 14.4, 14.1, 13.9, 13.8, 13.7. HRMS(+) calculated for $\left[\mathrm{C}_{58} \mathrm{H}_{80} \mathrm{~N}_{4} \mathrm{O}_{8} \mathrm{~S}_{5}\right] \mathrm{H}^{+}$: 1121.4653, found 1121.4639 .

Tetraethyl-5,5'-((1E,1'E)-((5,5'-((E)-ethene-1,2-diyl)bis(thiophene-5,2-diyl))bis(methanylylidene))bis(azanylylidene))bis(2-aminothiophene-3,4-dicarboxylate) (4). ${ }^{1} \mathrm{H}-\mathrm{NMR}\left(\mathrm{DMSO}-d_{6}\right): \delta=8.18$ (s, 2H), 7.95 (br, 4H), 7.51 (d, 2H, $\left.J=4.0 \mathrm{~Hz}\right), 7.31$ (d, $2 \mathrm{H}, J=4.0 \mathrm{~Hz}), 7.27(\mathrm{~s}, 2 \mathrm{H}), 4.27(\mathrm{q}, 4 \mathrm{H}, J=7.2 \mathrm{~Hz}), 4.14(\mathrm{q}, 4 \mathrm{H}, J=7.2 \mathrm{~Hz}), 1.34(\mathrm{t}$, $12 \mathrm{H}, J=7.0 \mathrm{~Hz}$ ). ${ }^{13} \mathrm{C}-\mathrm{NMR}\left(\mathrm{DMSO}-d_{6}\right): \delta=164.9,163.4,161.1,145.5,141.6,133.7$, 132.1, 129.5, 129.1, 125.1, 123.1, 100.4, 61.0, 59.8, 14.4, 14.3. HRMS(+) calculated for $\left[\mathrm{C}_{32} \mathrm{H}_{32} \mathrm{~N}_{4} \mathrm{O}_{8} \mathrm{~S}_{5}\right] \mathrm{H}^{+}:$729.1176, found 729.1160 . 

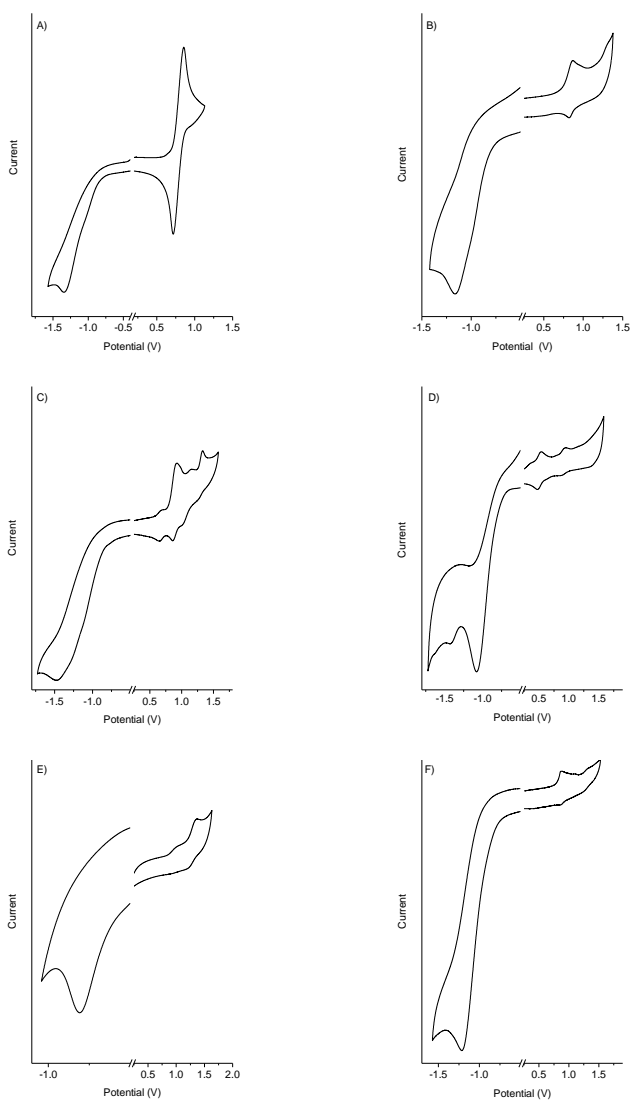

Figure S1. Cyclic voltammograms of 1B (A), 2A (B), 2B (C), 3A (D), 3B (E), and 4 (F) measured at $100 \mathrm{mV} / \mathrm{sec}$ in dichloromethane with $0.1 \mathrm{M} \mathrm{TBAPF}_{6} \mathrm{Vs} . \mathrm{Ag}^{0}$. 

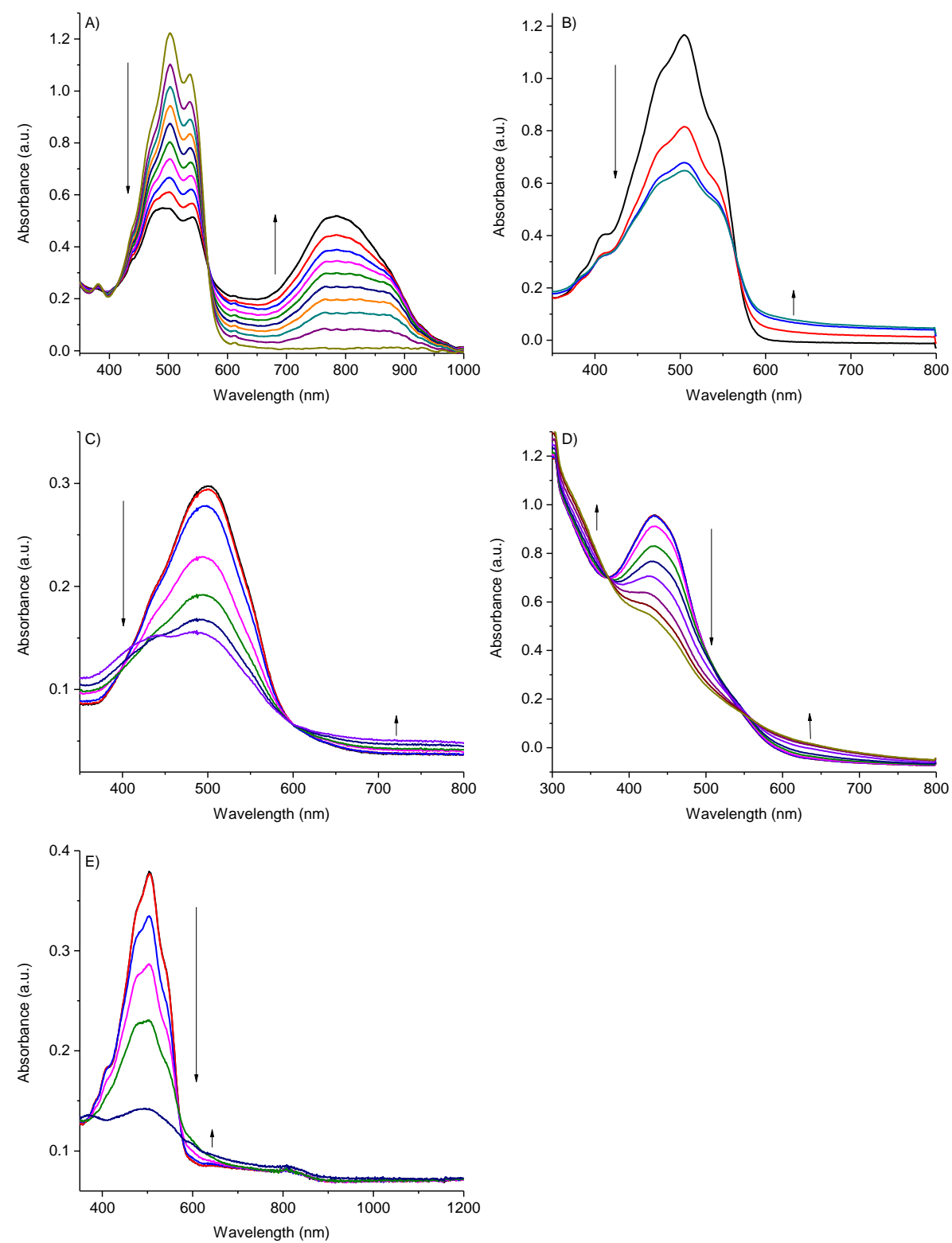

Figure S2. Spectroelectrochemistry measured in $0.1 \mathrm{M} \mathrm{TBAPF}_{6}$ in dichloromethane: A) $\mathbf{1 B}$ from $0 \mathrm{mV}$ to $900 \mathrm{mV}$, B) $\mathbf{2 A}$ from $0 \mathrm{mV}$ to $1.0 \mathrm{~V}$, C) $\mathbf{3 A}$ between $0 \mathrm{mV}$ and $1.5 \mathrm{~V}$, D) $3 \mathrm{~B}$ between $0 \mathrm{mV}$ and $1.2 \mathrm{~V}$, and E) 4 between $0 \mathrm{mV}$ and $1.1 \mathrm{~V}$. 


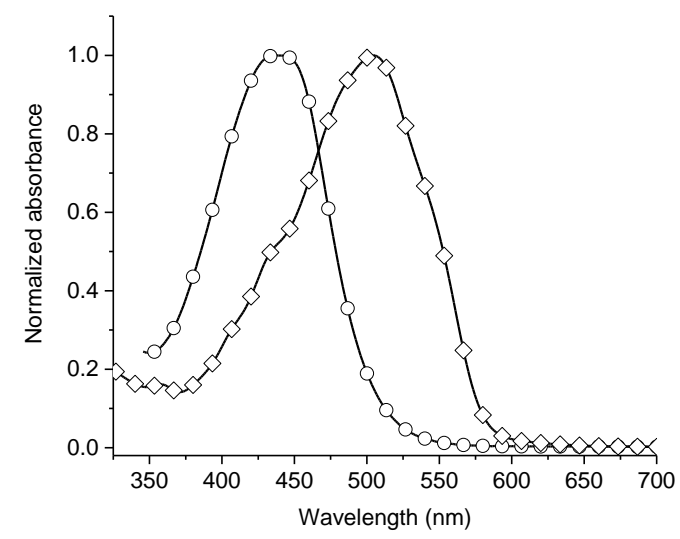

Figure S3. Normalized absorption spectra of $\mathbf{2 B}(\bigcirc)$ and $\mathbf{3 A}(\diamond)$.

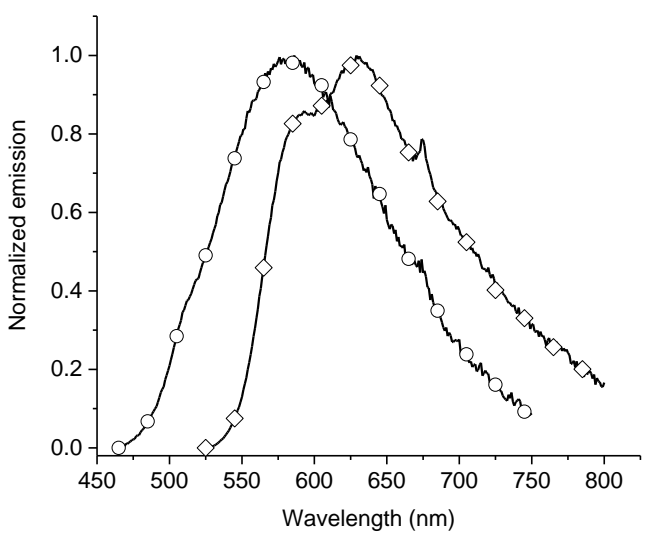

Figure S4. Normalized emission spectra of $\mathbf{2 B}(\bigcirc)$ and $\mathbf{3 A}(\diamond)$.

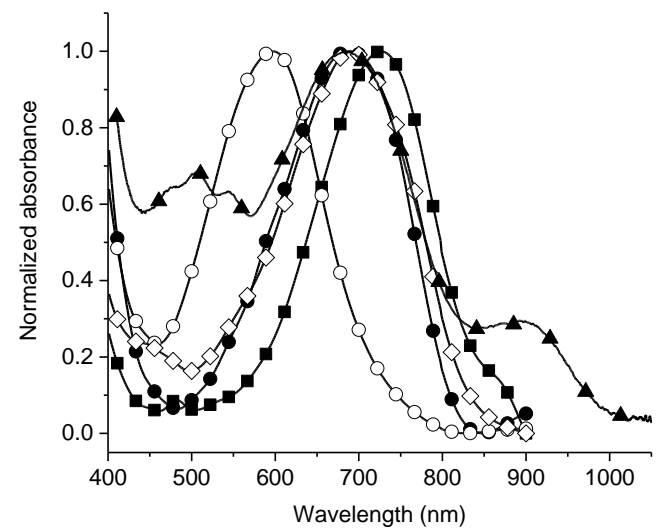

Figure S5. Normalized absorption spectra of $\mathbf{1 B}(\bullet), \mathbf{2 A}(\bullet), \mathbf{2 B}(\circ), \mathbf{3 A}(\diamond), \mathbf{3 B}(\diamond)$, and $4(\Delta)$ in dichloromethane with $\mathrm{FeCl}_{3}$ added. 

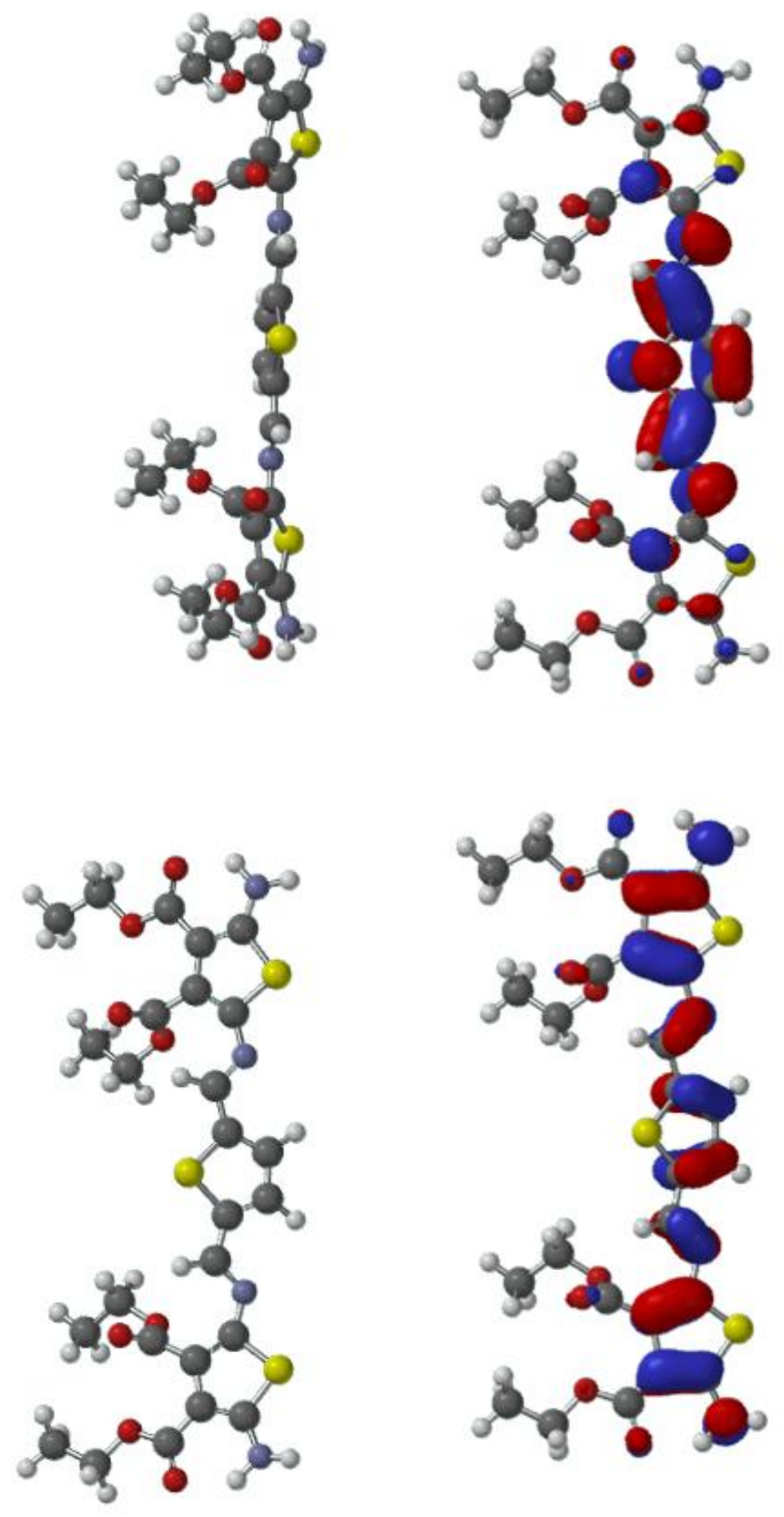

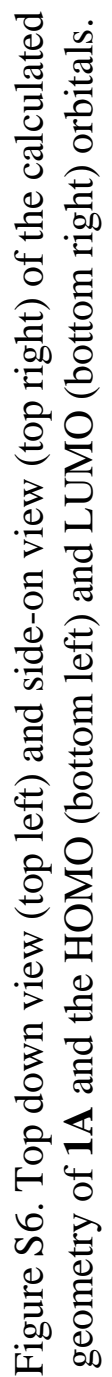



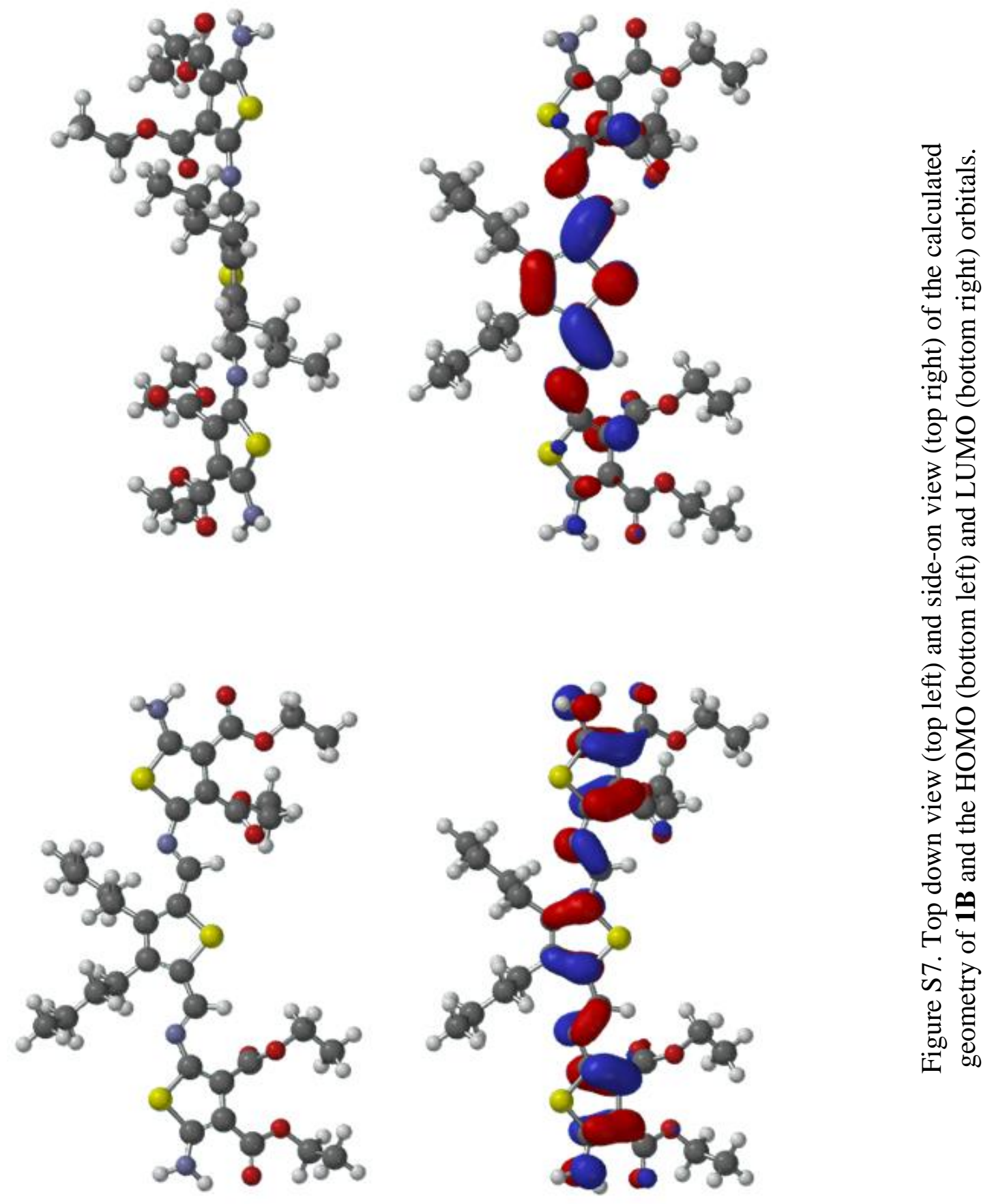

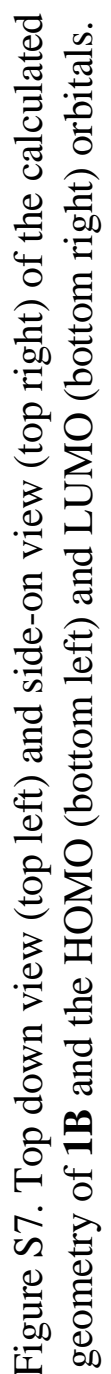

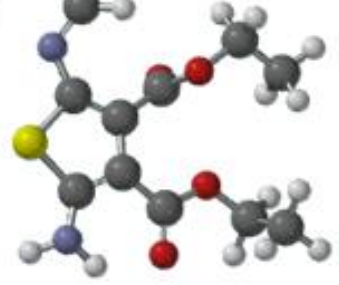



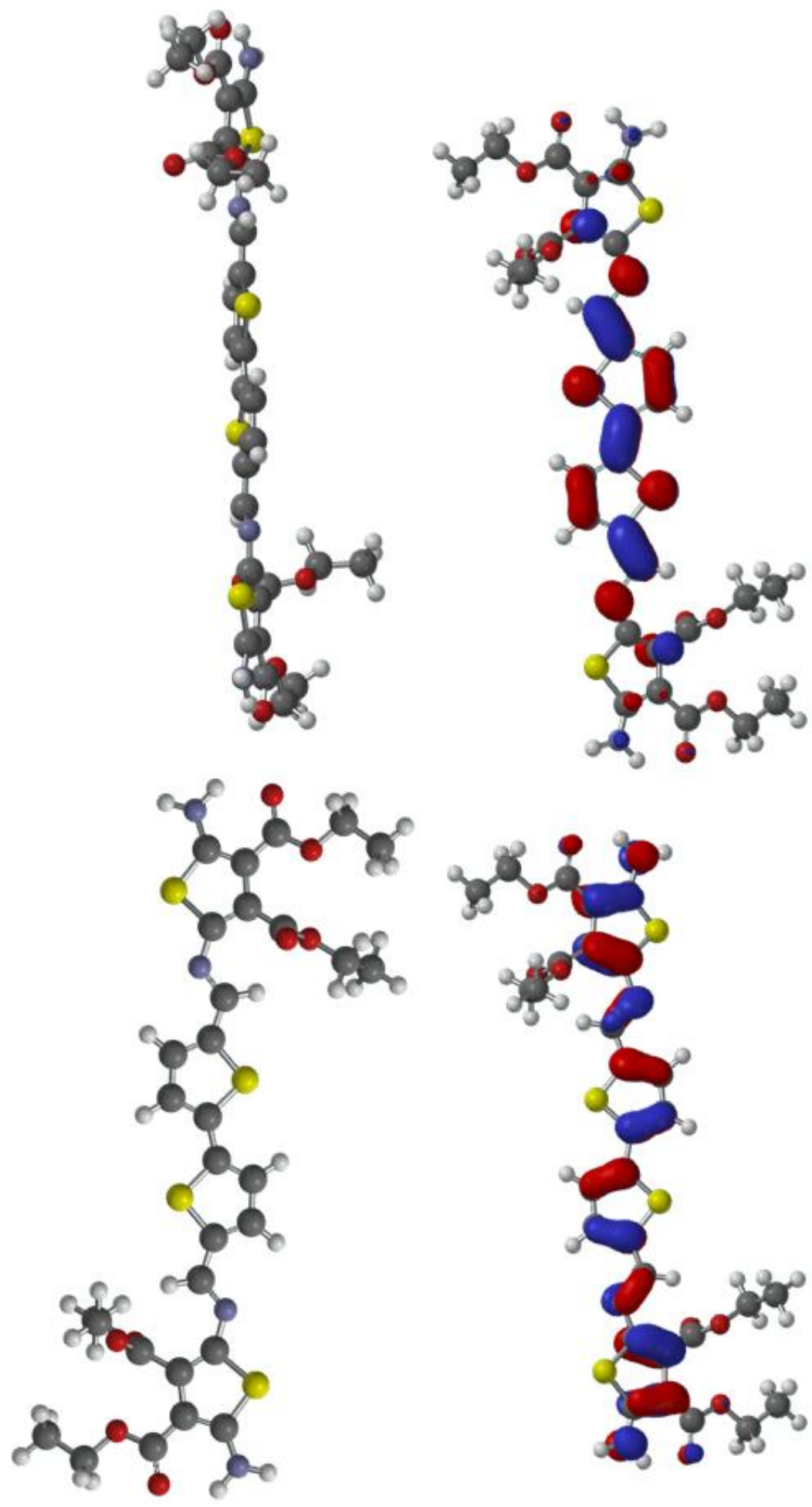

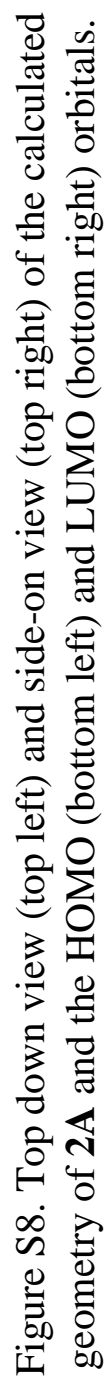

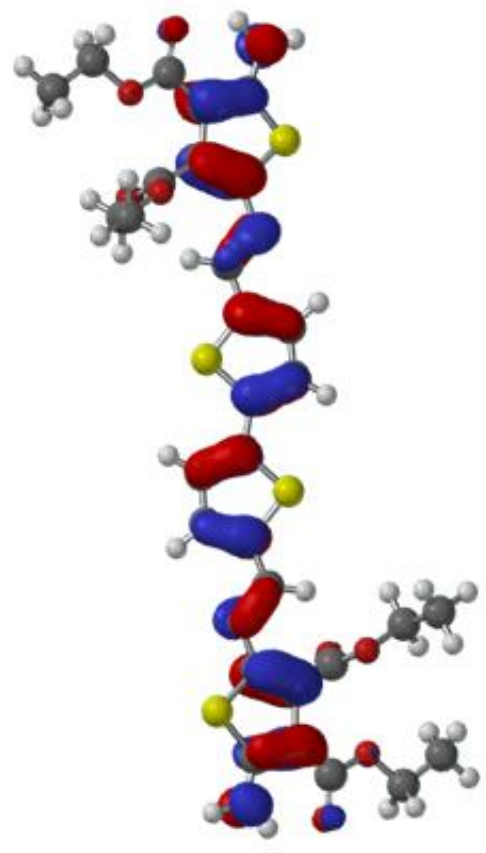



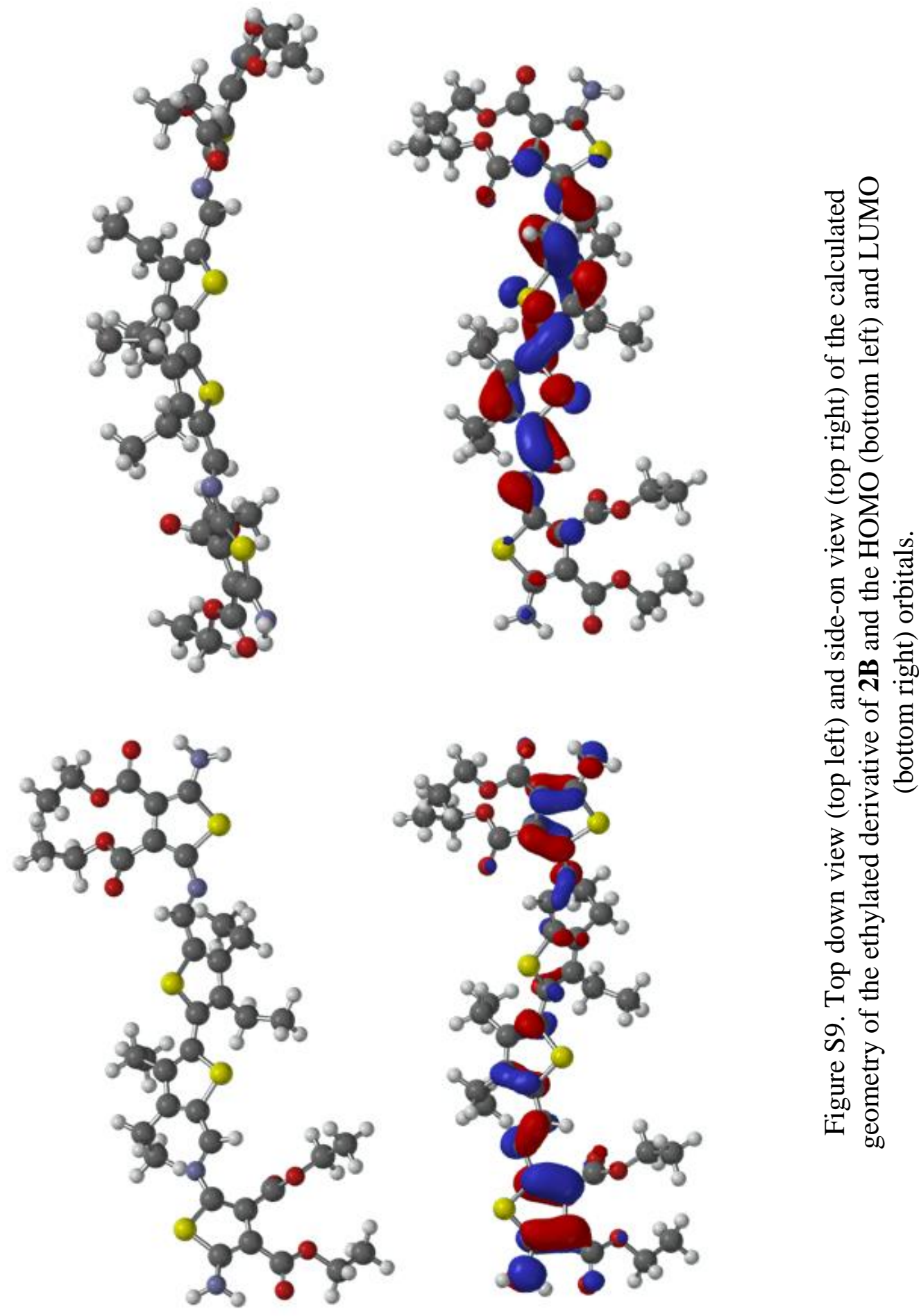


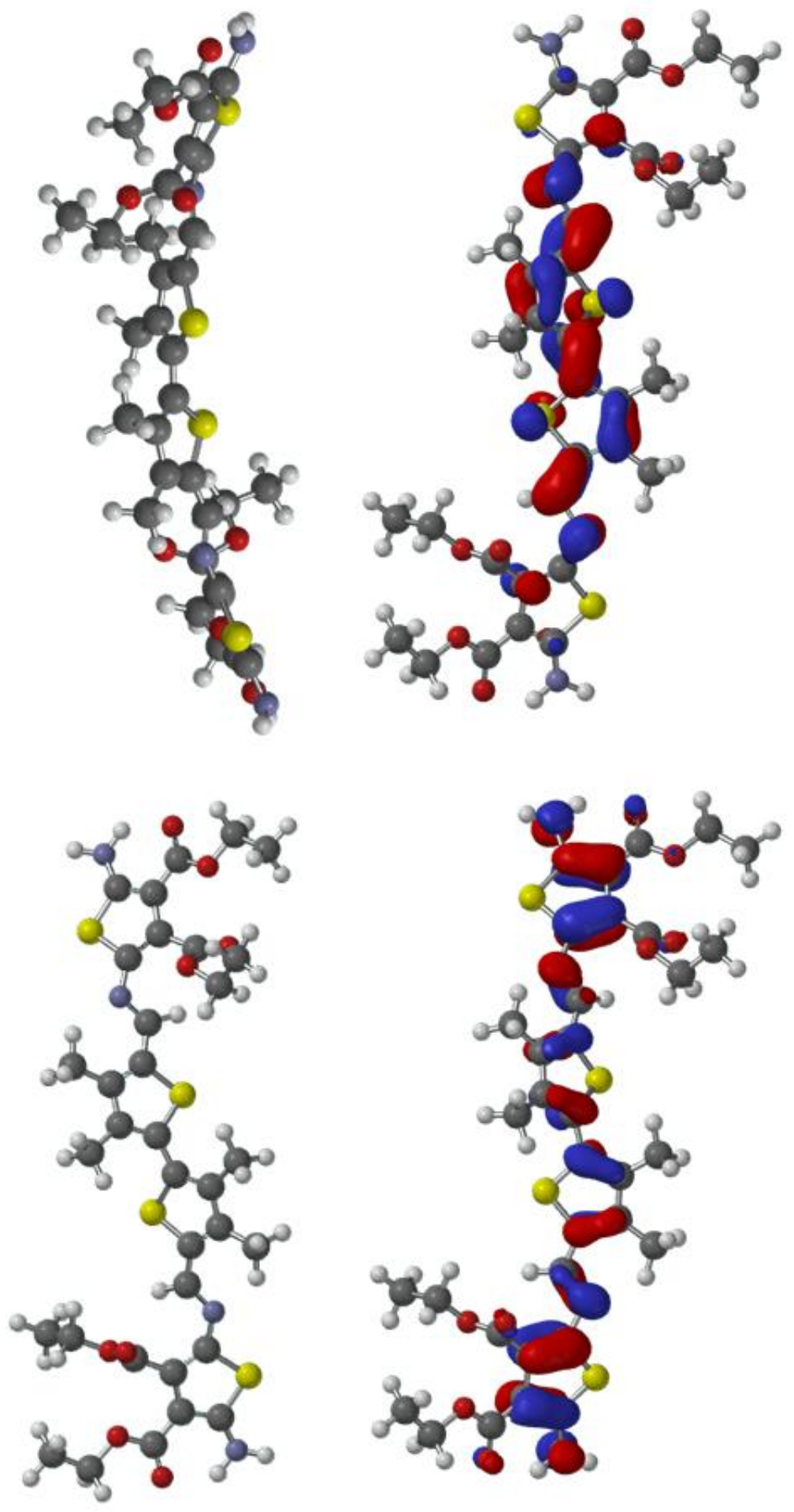

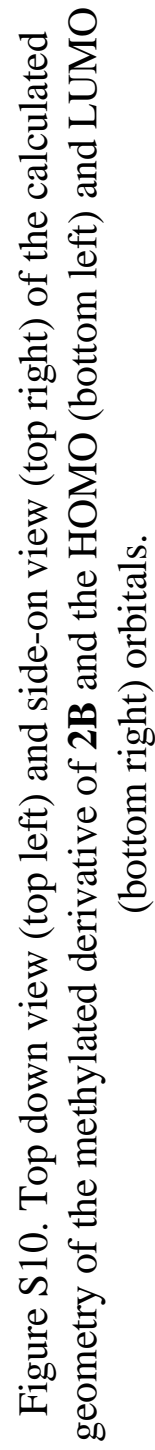



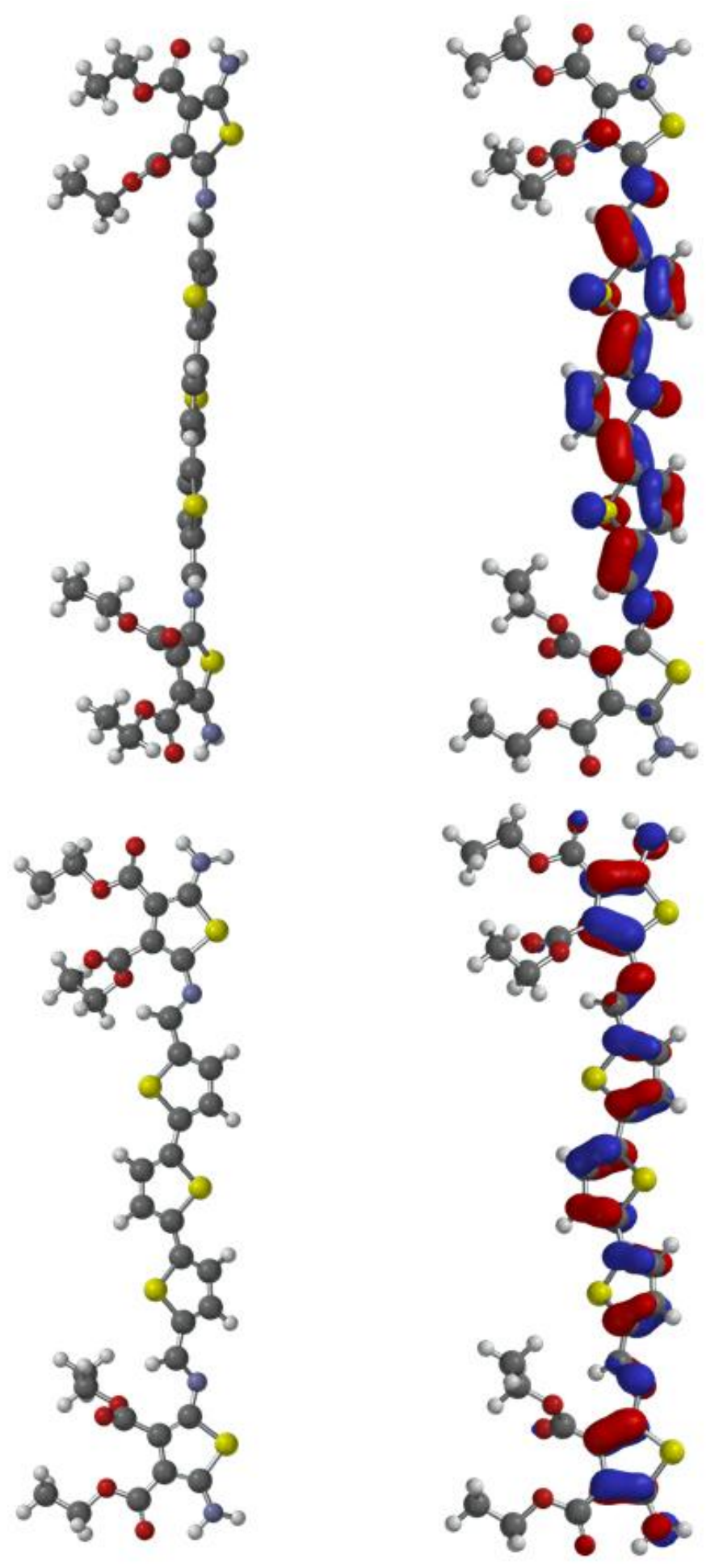

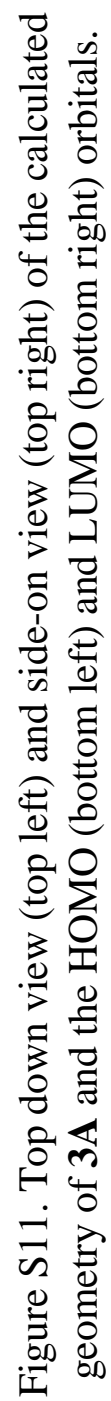



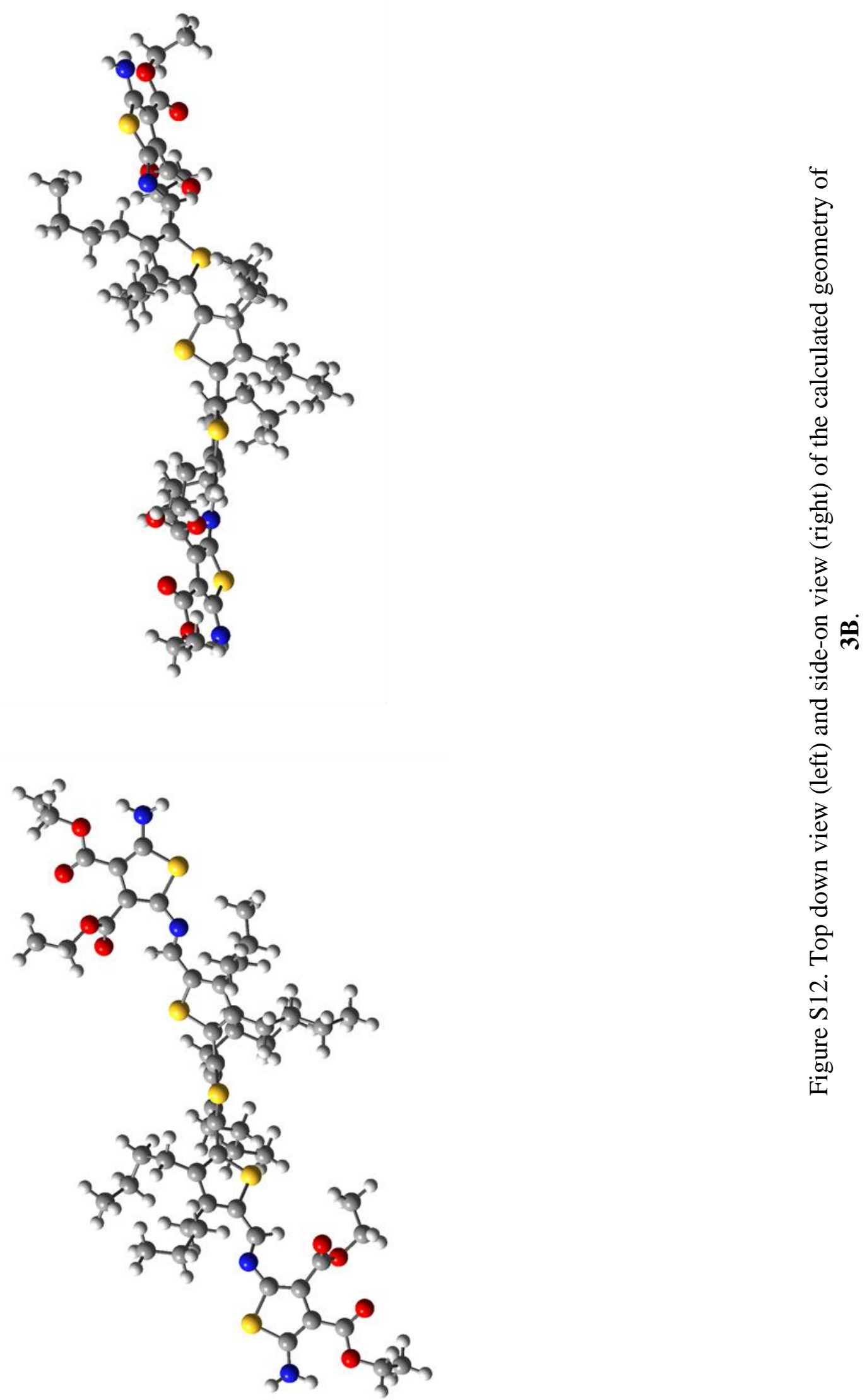

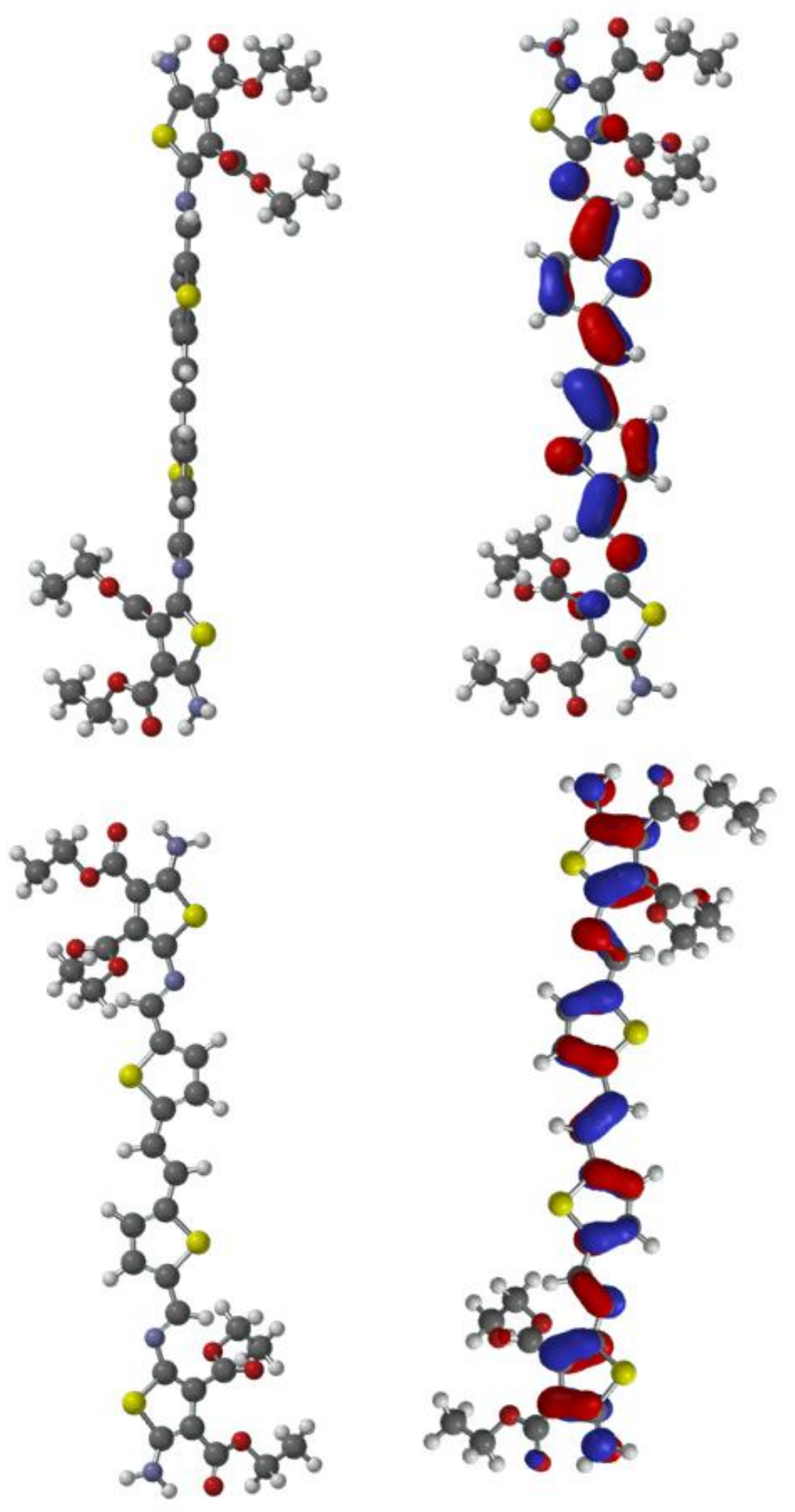

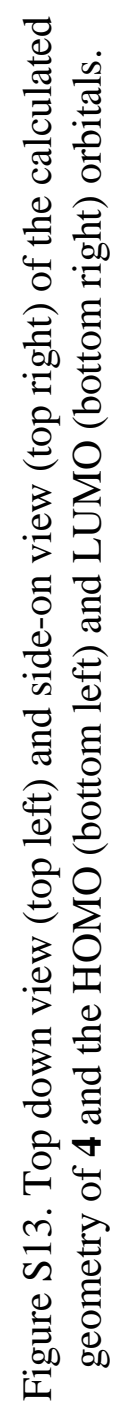




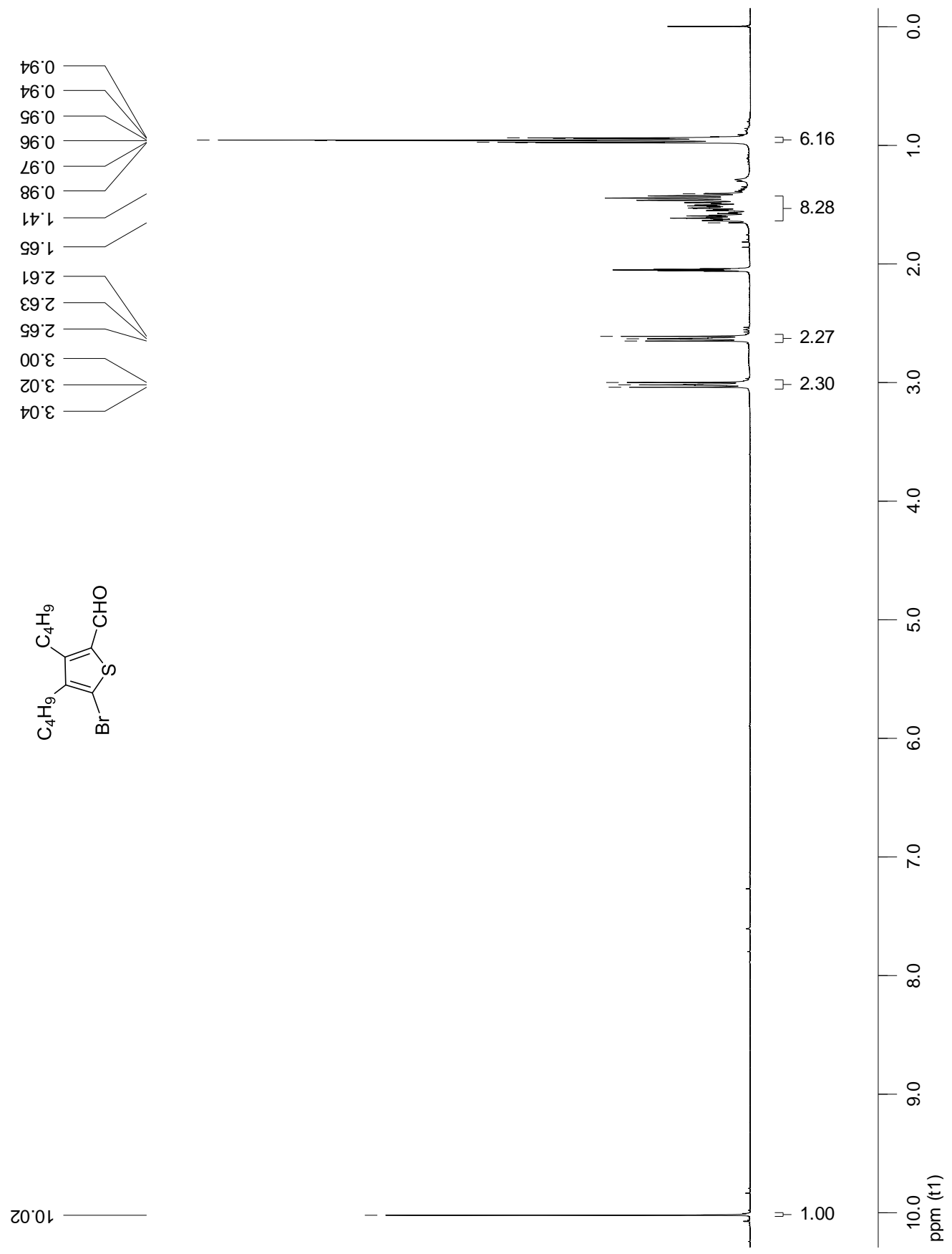

Figure S14. ${ }^{1} \mathrm{H}$ NMR spectrum of 5-bromo-3,4-dibutylthiophene-2-carboxaldehyde recorded in acetone- $\mathrm{d}_{6}$. 


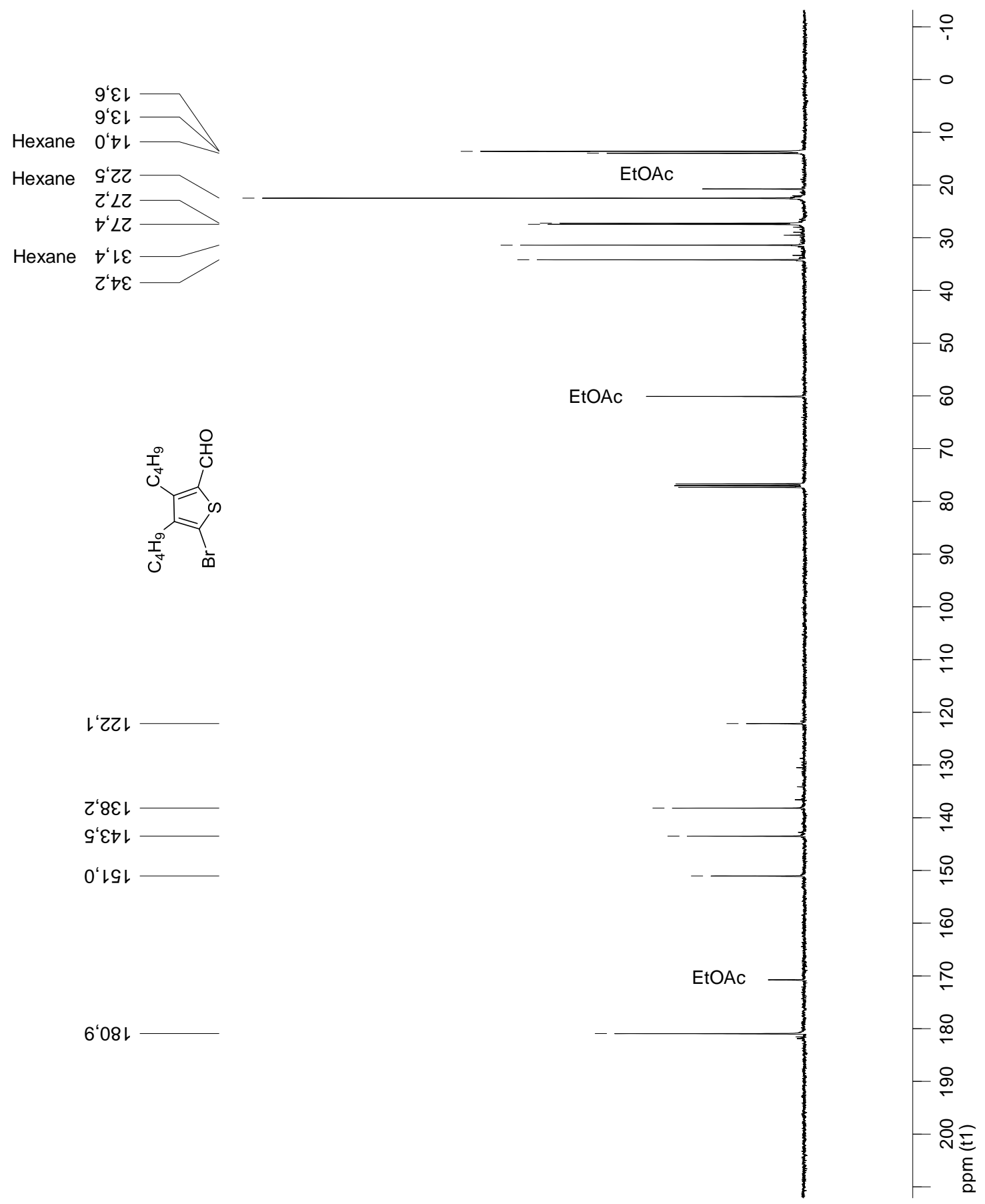

Figure S15. ${ }^{13} \mathrm{C}$ NMR spectrum of 5-bromo-3,4-dibutylthiophene-2-carboxaldehyde recorded in $\mathrm{CDCl}_{3}$. 


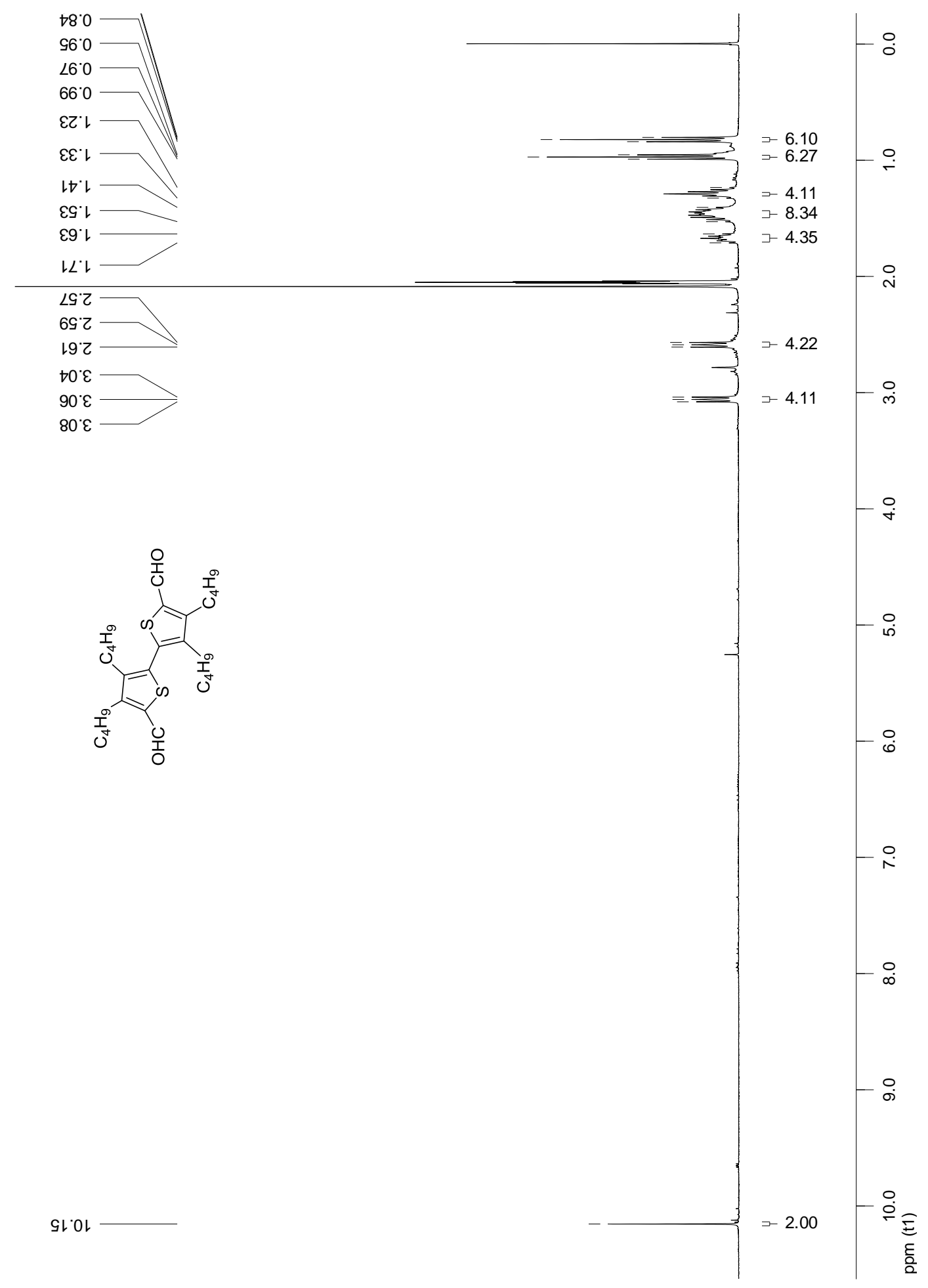

Figure S16. ${ }^{1} \mathrm{H} \quad \mathrm{NMR}$ spectrum of 3,3',4,4'-tetrabutyl-[2,2'-bithiophene]-5,5'dicarboxaldehyde recorded in acetone- $\mathrm{d}_{6}$. 

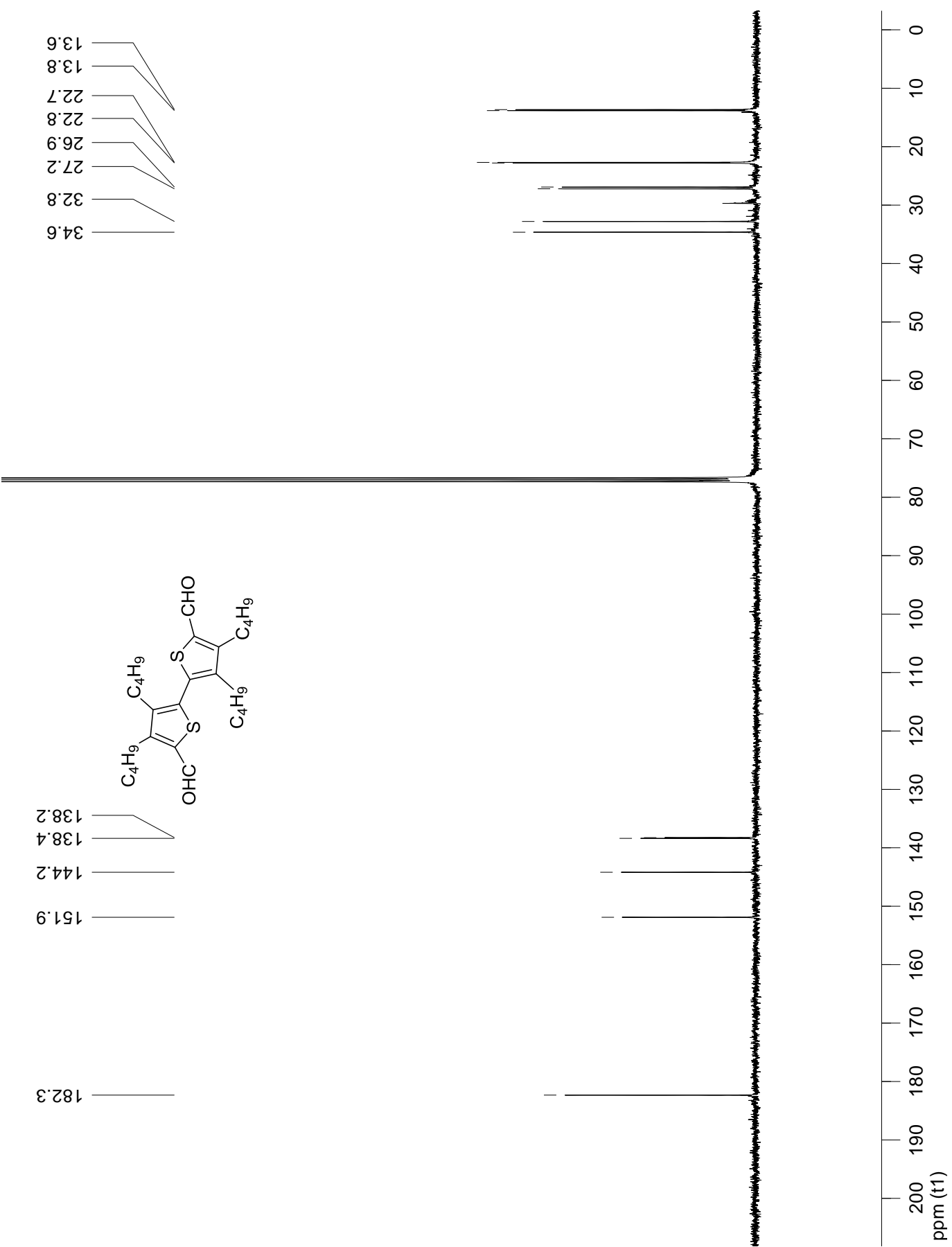

Figure S17. ${ }^{13} \mathrm{C}$ NMR spectrum of 3,3',4,4'-tetrabutyl-[2,2'-bithiophene]-5,5'dicarboxaldehyde recorded in $\mathrm{CDCl}_{3}$. 


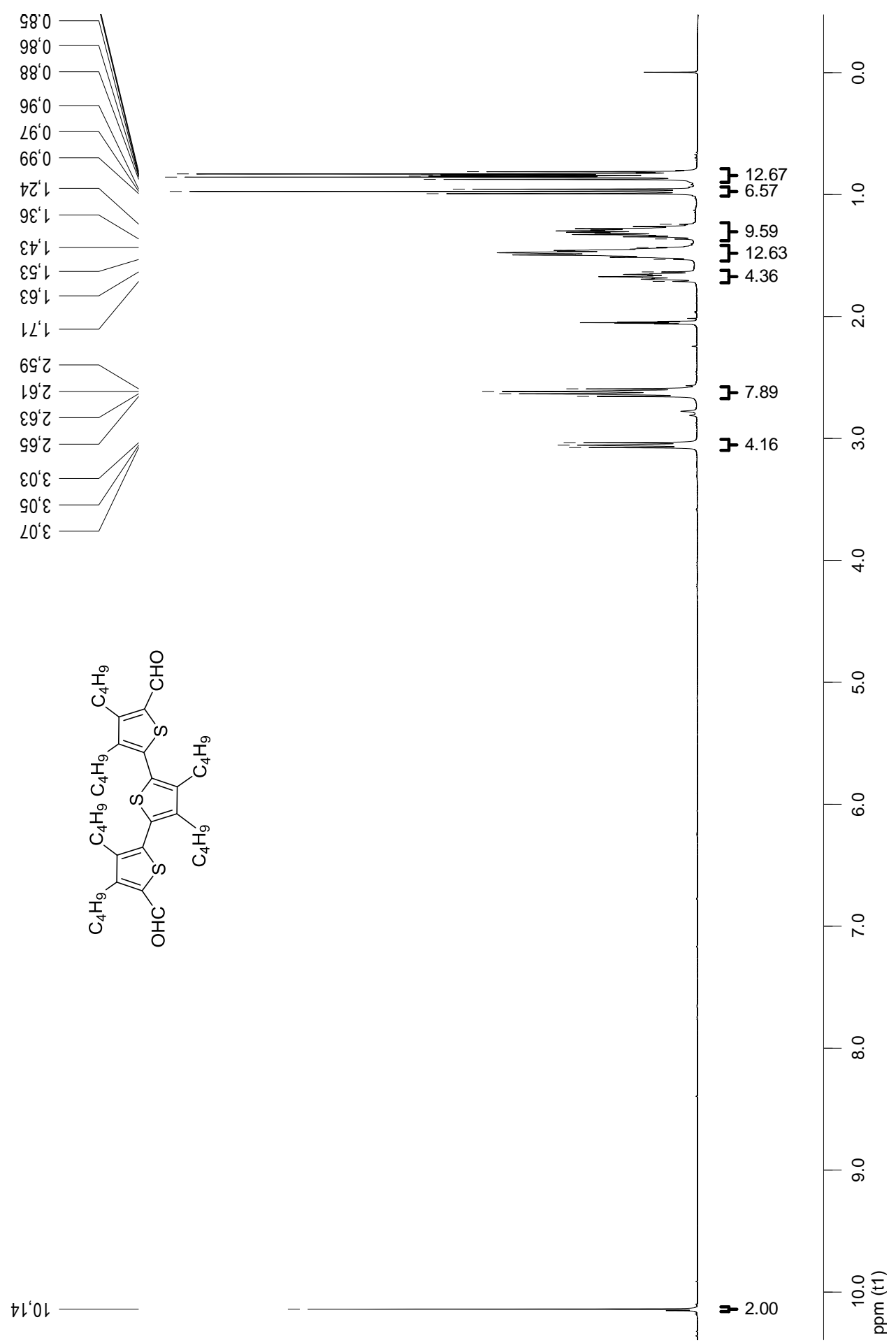

Figure S18. ${ }^{1} \mathrm{H}$ NMR spectrum of 3,3',3",4,4',4"-hexabutyl-[2,2':5',2"-terthiophene]-5,5"dicarboxaldehyde recorded in acetone- $\mathrm{d}_{6}$. 


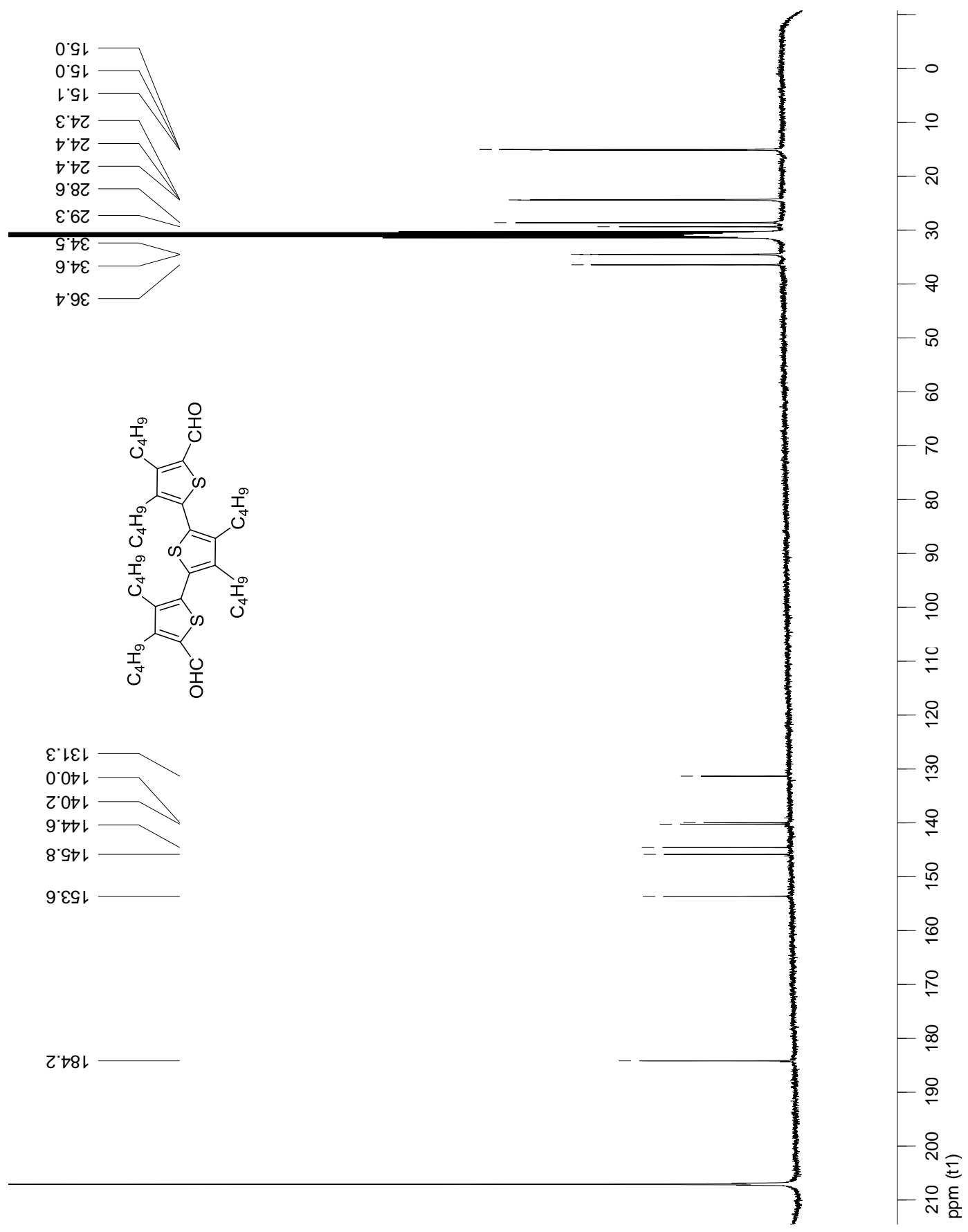

Figure S19. ${ }^{13} \mathrm{C}$ NMR spectrum of 3,3',3",4,4',4"-hexabutyl-[2,2':5',2"-terthiophene]-5,5"dicarboxaldehyde recorded in acetone- $\mathrm{d}_{6}$. 


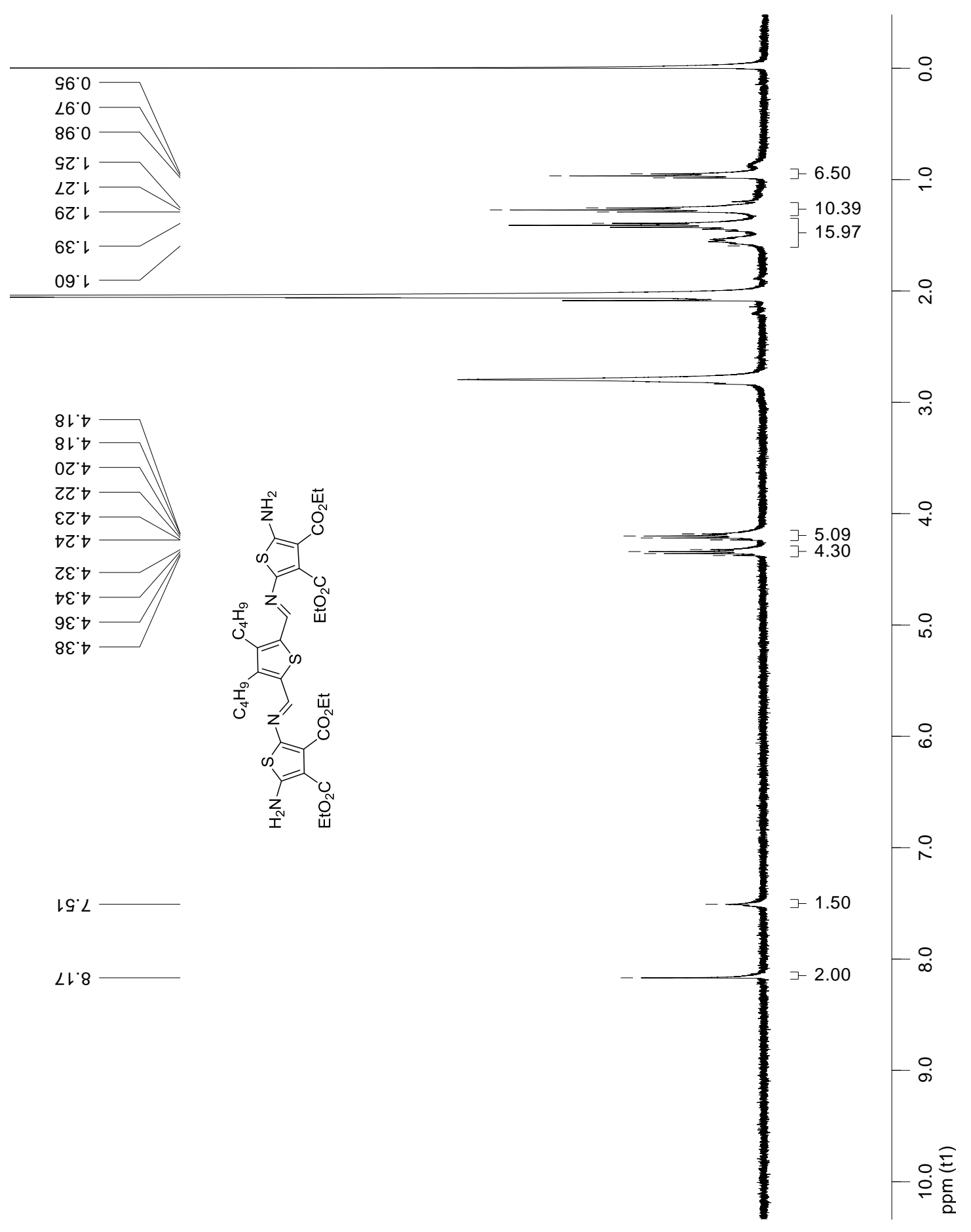

Figure S20. ${ }^{1} \mathrm{H}$ NMR spectrum of $\mathbf{1 B}$ recorded in acetone- $\mathrm{d}_{6}$. 


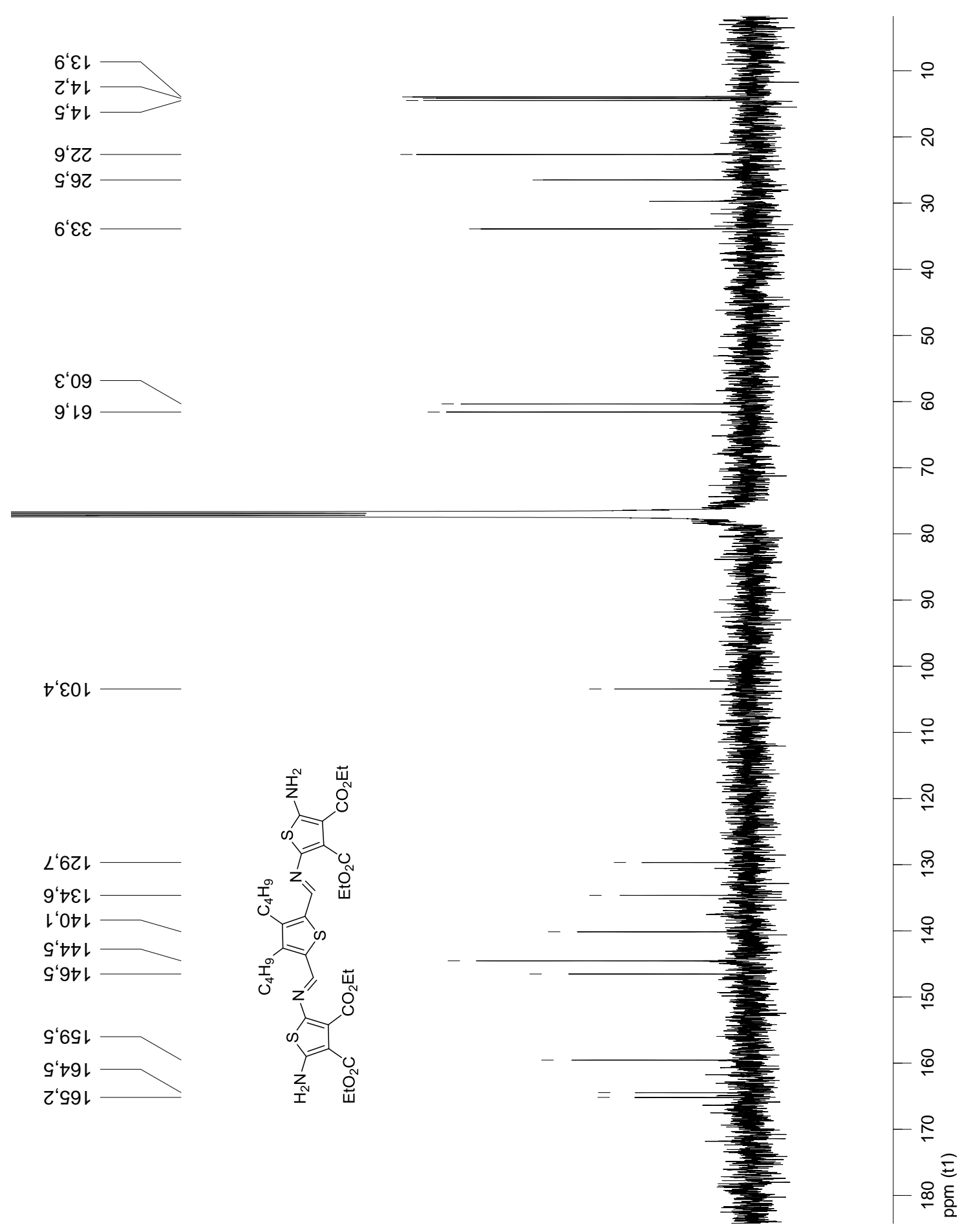

Figure S21. ${ }^{13} \mathrm{C}$ NMR spectrum of $\mathbf{1 B}$ recorded in $\mathrm{CDCl}_{3}$. 


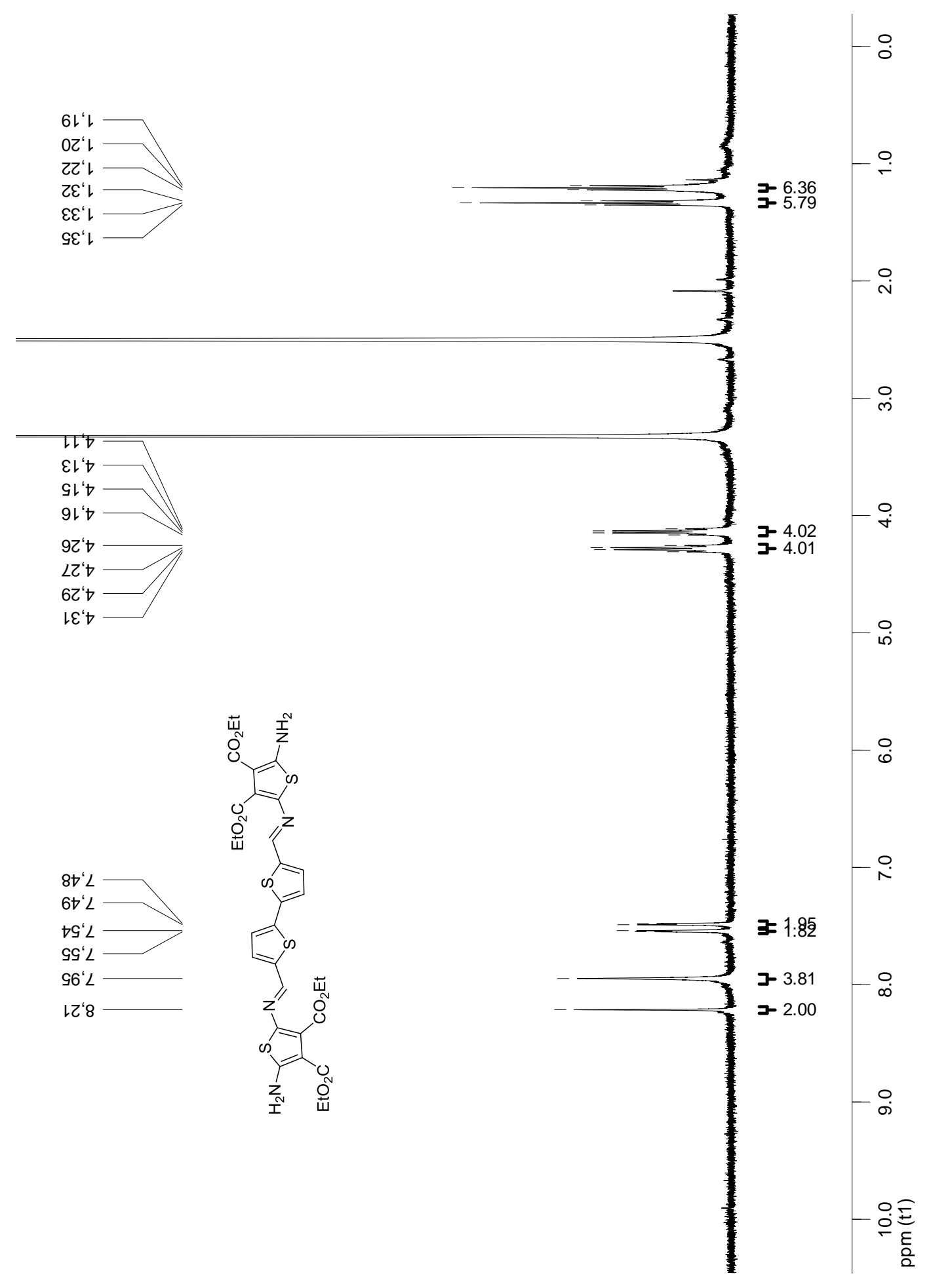

Figure S22. ${ }^{1} \mathrm{H}$ NMR spectrum of $\mathbf{2 A}$ recorded in DMSO-d $\mathrm{d}_{6}$. 


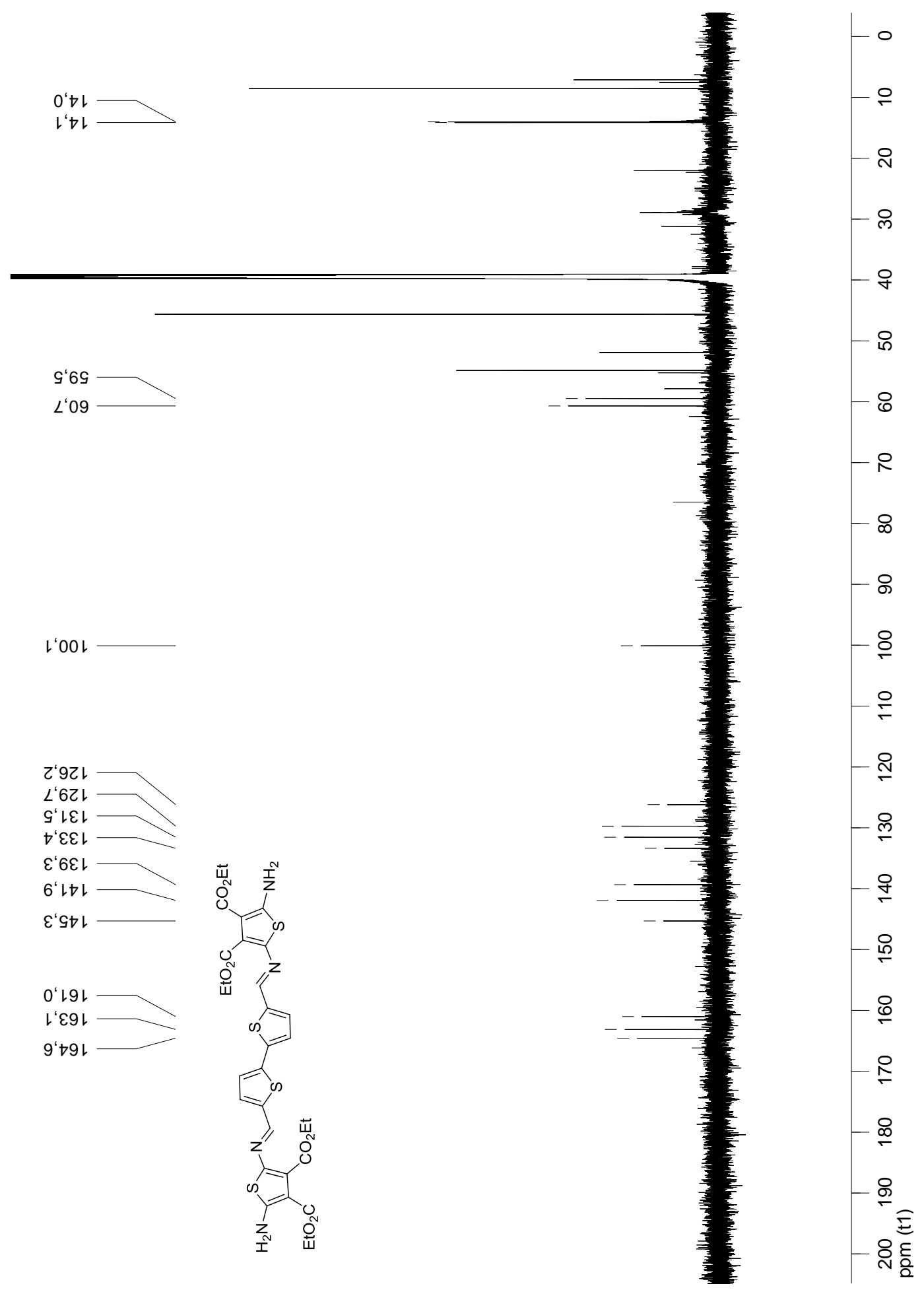

Figure S23. ${ }^{13} \mathrm{C}$ NMR spectrum of $\mathbf{2 A}$ recorded in DMSO-d $\mathrm{d}_{6}$. 


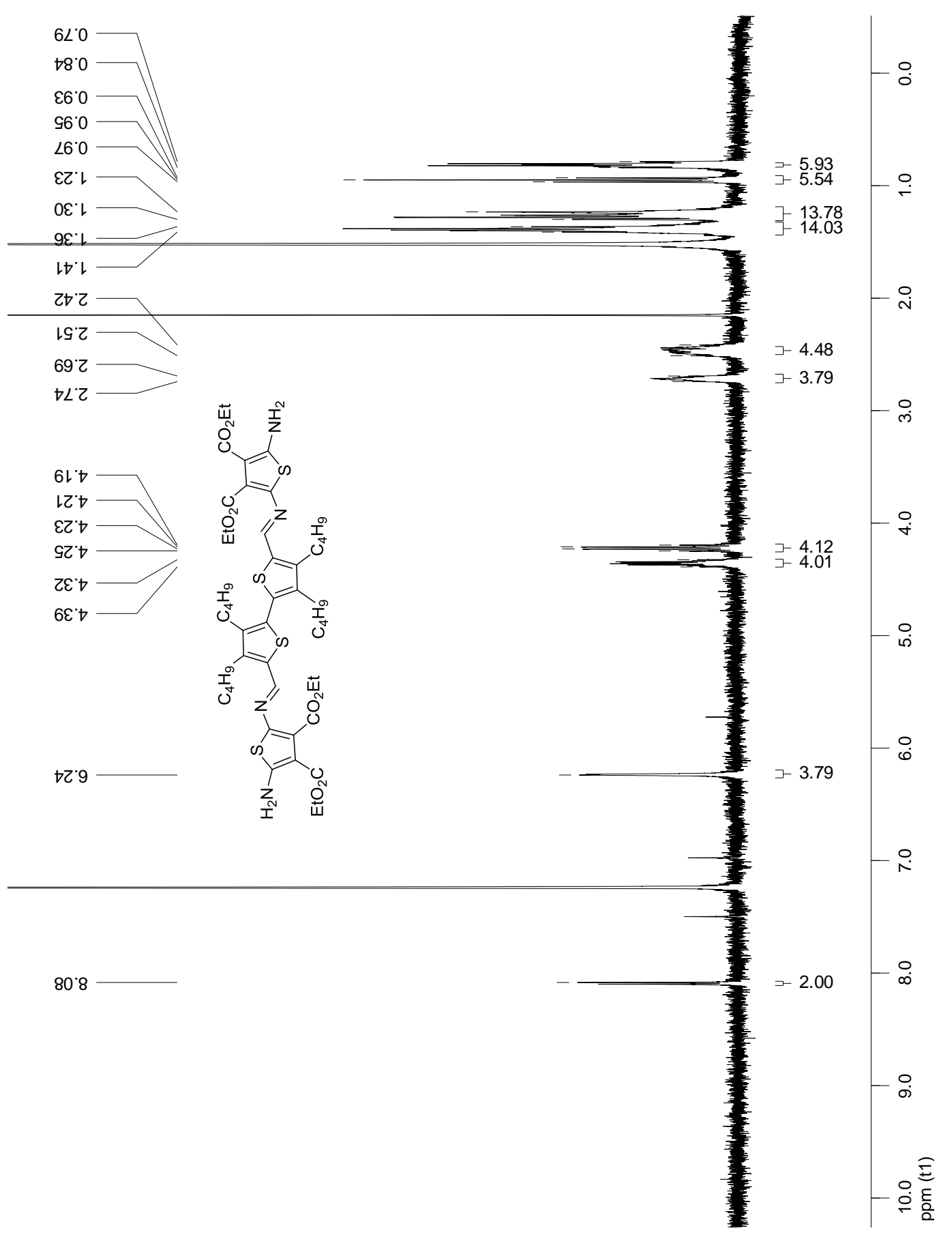

Figure S24. ${ }^{1} \mathrm{H}$ NMR spectrum of $\mathbf{2 B}$ recorded in $\mathrm{CDCl}_{3}$. 


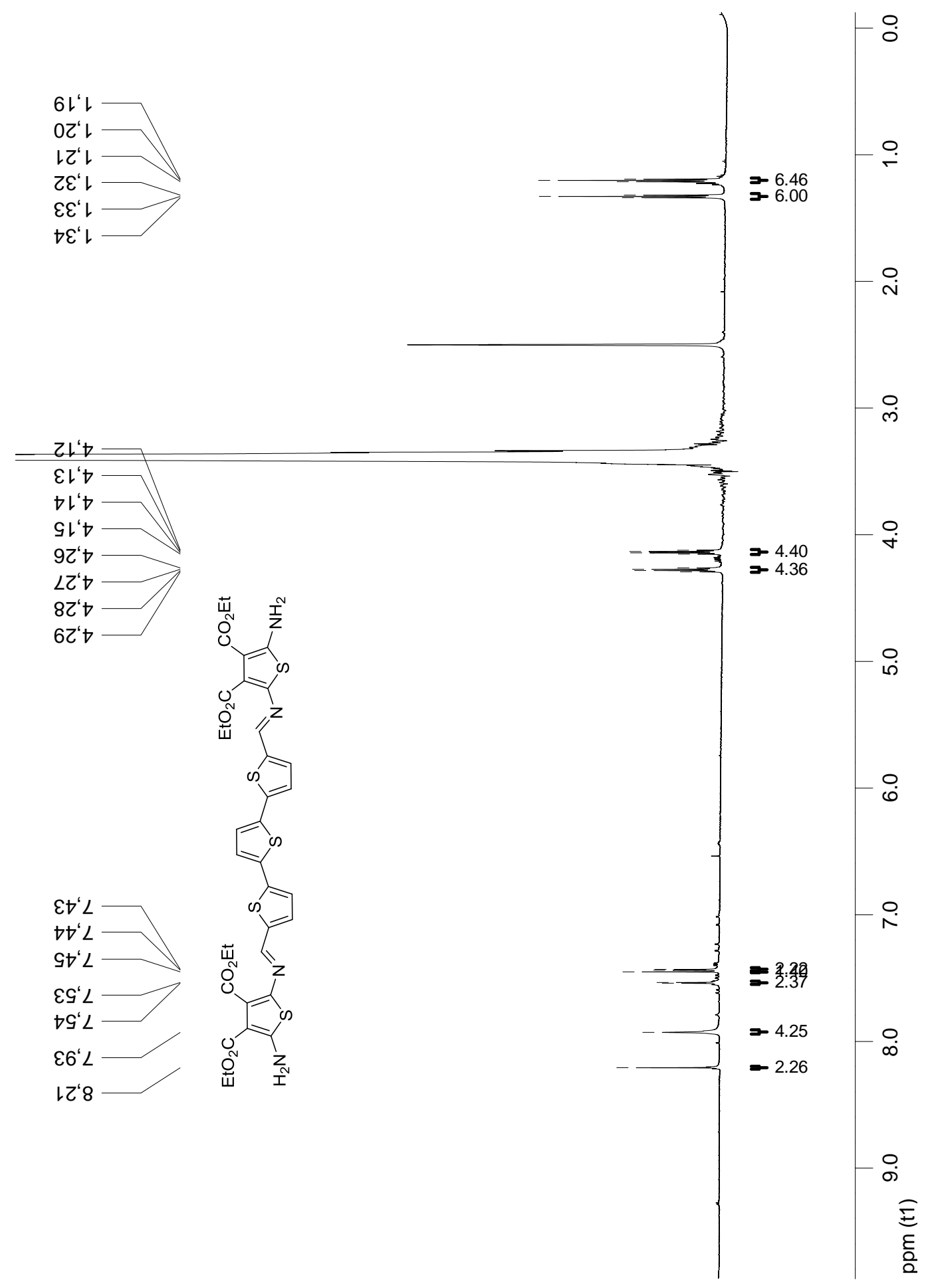

Figure S25. ${ }^{1} \mathrm{H}$ NMR spectrum of $\mathbf{3 A}$ recorded in DMSO-d $\mathrm{d}_{6}$. 


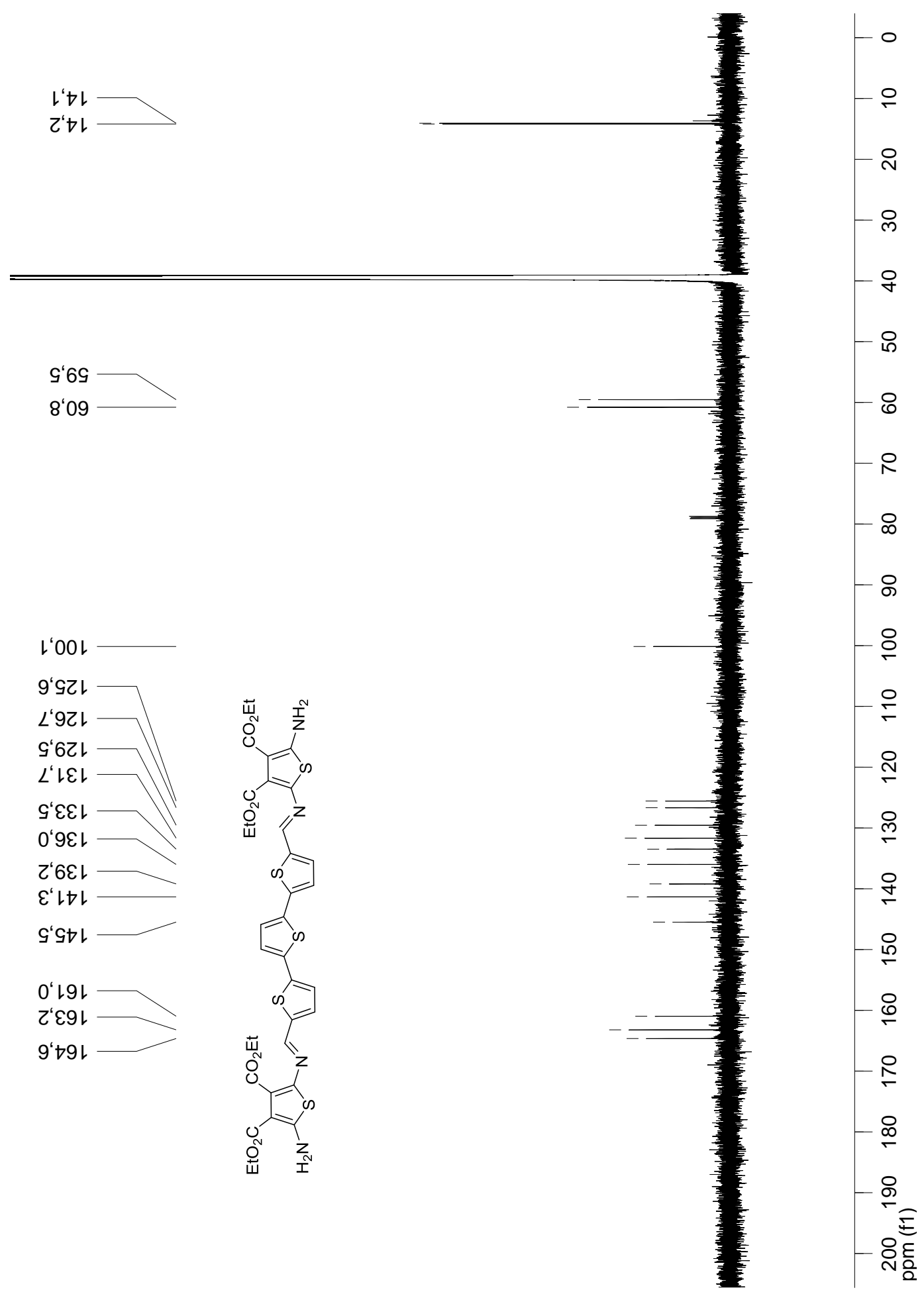

Figure S26. ${ }^{13} \mathrm{C}$ NMR spectrum of $\mathbf{3 A}$ recorded in DMSO- $\mathrm{d}_{6}$. 


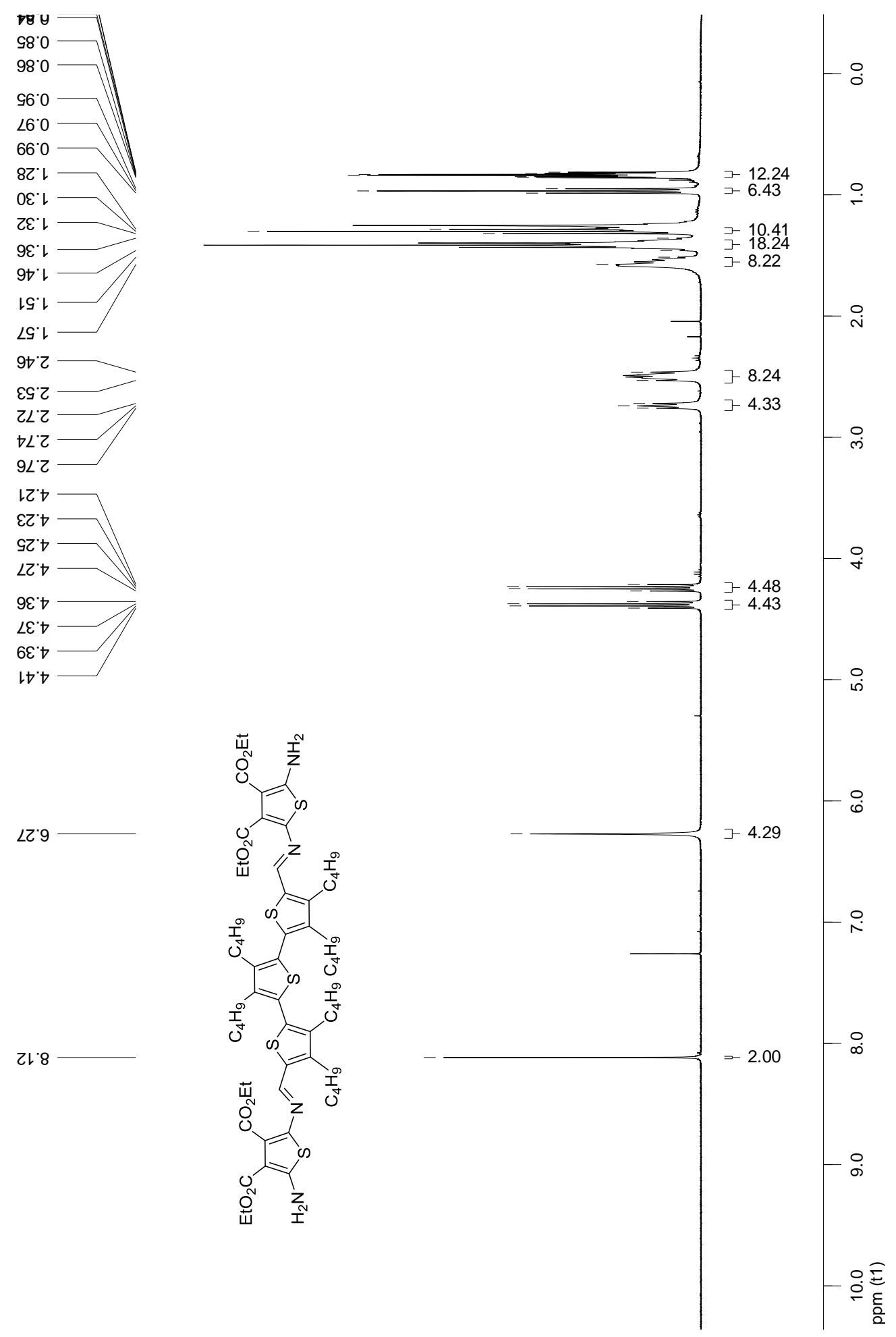

Figure S27. ${ }^{1} \mathrm{H}$ NMR spectrum of $\mathbf{3 B}$ recorded in $\mathrm{CDCl}_{3}$. 


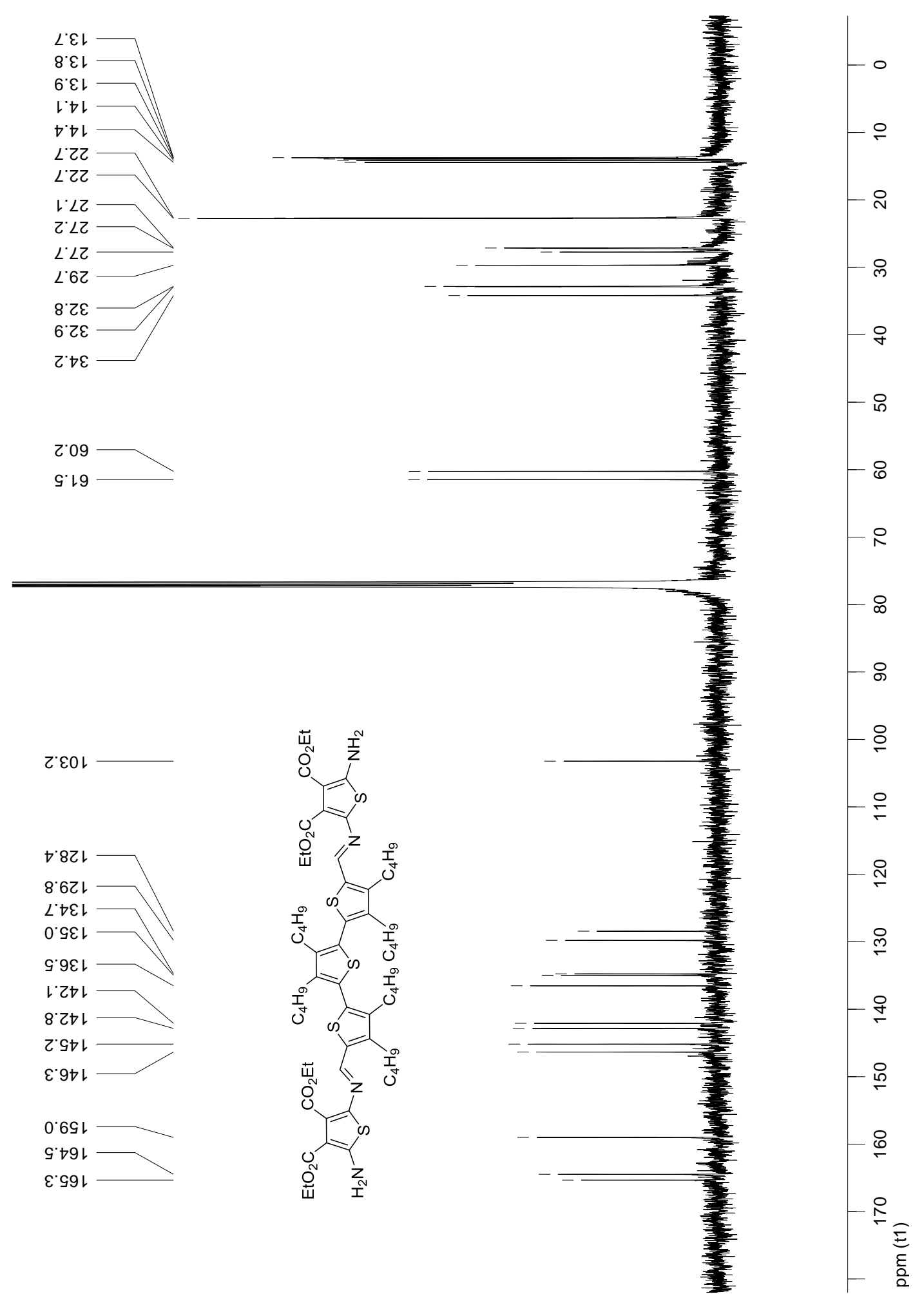

Figure S28. ${ }^{13} \mathrm{C}$ NMR spectrum of $\mathbf{3 B}$ recorded in $\mathrm{CDCl}_{3}$. 


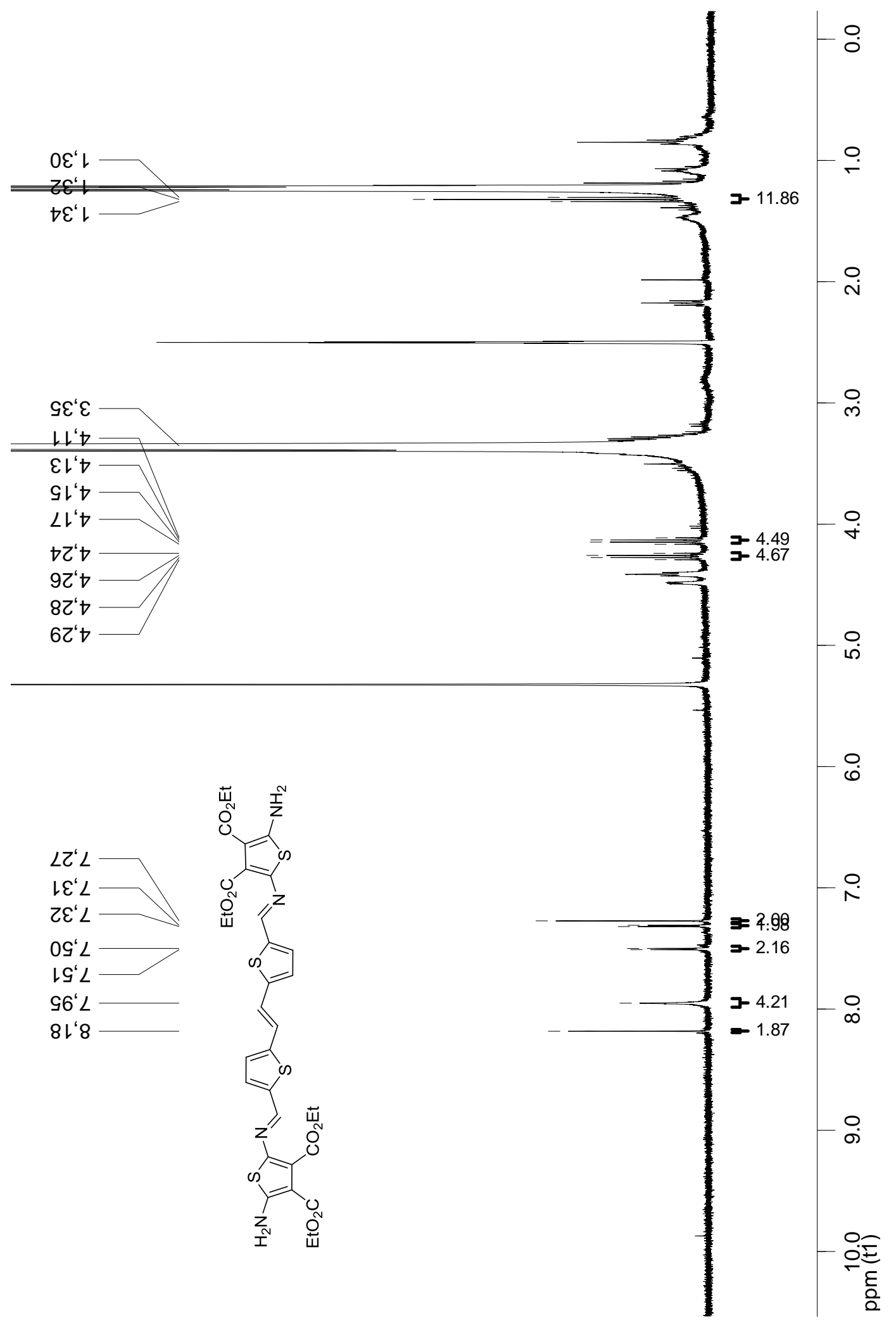

Figure S29. ${ }^{1} \mathrm{H}$ NMR spectrum of $\mathbf{4}$ recorded in DMSO-d 6 . 


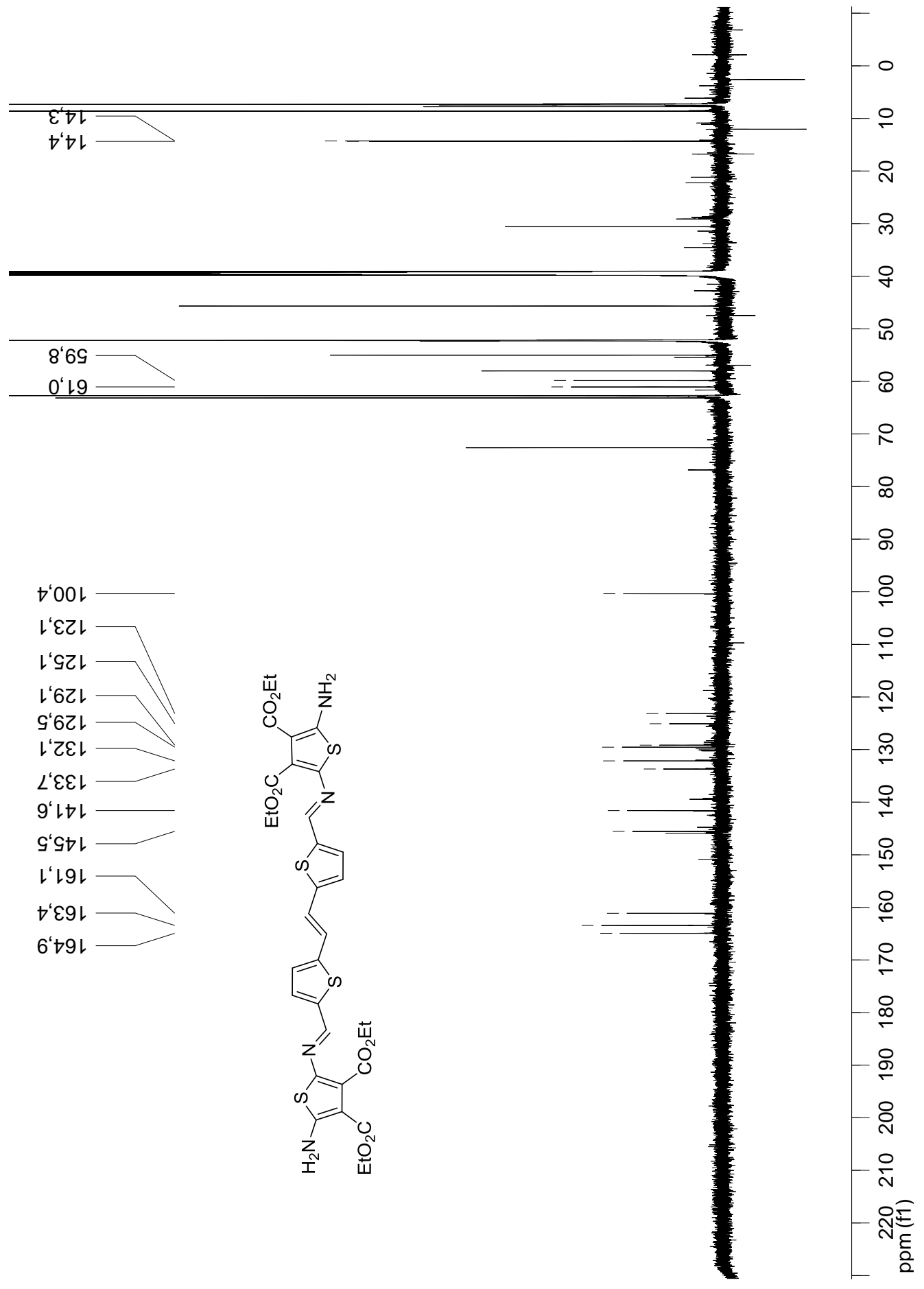

Figure S30. ${ }^{13} \mathrm{C}$ NMR spectrum of $\mathbf{4}$ recorded in DMSO-d $\mathrm{d}_{6}$. 


\section{References}

(1) Connelly, N. G.; Geiger, W. E., Chemical Redox Agents for Organometallic Chemistry. Chem. Rev. 1996, 96, 877-910.

(2) Bishop, S. L'impact de l'Unité Centrale Sur les Propriétés Optoélectroniques d'Imino-Thiophènes Électrochromes : Synthèse et Caractérisation. M. Sc. Thesis, Université de Montréal, Montreal, QC, 2013.

(3) Agrawal, A. K.; Jenekhe, S. A., Electrochemical Properties and Electronic Structures of Conjugated Polyquinolines and Polyanthrazolines. Chem. Mater. 1996, 8, 579-589.

(4) Frisch, M. J., et al. Gaussian 09, Revision D.01, Gaussian, Inc.: Wallingford CT, 2009.

(5) Becke, A. D., Density-Functional Thermochemistry. III. The Role of Exact Exchange. J. Chem. Phys. 1993, 98, 5648-5652.

(6) Ditchfield, R.; Hehre, W. J.; Pople, J. A., Self-Consistent Molecular-Orbital Methods.

IX. An Extended Gaussian-Type Basis for Molecular-Orbital Studies of Organic

Molecules. J. Chem. Phys. 1971, 54, 724-728.

(7) Hehre, W. J.; Ditchfield, R.; Pople, J. A., Self-Consistent Molecular Orbital Methods. XII. Further Extensions of Gaussian-Type Basis Sets for Use in Molecular Orbital Studies of Organic Molecules. J. Chem. Phys. 1972, 56, 2257-2261.

(8) Hariharan, P. C.; Pople, J. A., Accuracy of $\mathrm{AH}_{\mathrm{n}}$ Equilibrium Geometries by Single Determinant Molecular Orbital Theory. Mol. Phys. 1974, 27, 209-214.

(9) Widom, A.; Clark, T. D.; Megaloudis, G., Higher Harmonics in the Josephson Effect. Phys. Lett. A 1980, 76, 163-164.

(10) Gewald, V. K.; Kleinert, M.; Thiele, B.; Hentschel, M., zur Basenkatalysierten Reaktion von Methylenaktiven Nitrilen mit Schwefel. J. Prak. Chem. 1972, 314, 303314.

(11) Elandaloussi, E. H.; Frère, P.; Richomme, P.; Orduna, J.; Garin, J.; Roncali, J., Effect of Chain Extension on the Electrochemical and Electronic Properties of $\pi$ Conjugated Soluble Thienylenevinylene Oligomers. J. Am. Chem. Soc. 1997, 119, 1077410784.

(12) Starčević, K.; Boykin, D. W.; Karminski-Zamola, G., New Amidino-Benzimidazolyl Thiophenes: Synthesis and Photochemical Synthesis. Heteroatom Chem. 2003, 14, 218 222.

(13) Piron, F.; Leriche, P.; Grosu, I.; Roncali, J., Electropolymerizable 3D $\pi$-Conjugated Architectures with Ethylenedioxythiophene (EDOT) End-Groups as Precursors of Electroactive Conjugated Networks. J. Mater. Chem. 2010, 20, 10260-10268.

(14) Nakayama, J.; Fujimori, T., An Improved One-Pot Synthesis of 2,2'-Bithiophene5,5'-Dicarboxaldehyde and 2,2':5',2"-Terthiophene-5,5"-Dicarbaldehyde. Sulfur Lett. 1990, 11, 29-30.

(15) Bader, M. M.; Pham, P.-T. T.; Elandaloussi, E. H., Dicyanovinyl-Substituted Oligothiophenes. Cryst. Growth Des. 2010, 10, 5027-5030.

(16) Dufresne, S.; Bolduc, A.; Skene, W. G., Towards Materials with Reversible Oxidation and Tuneable Colours Using Heterocyclic Conjugated Azomethines. J. Mater. Chem. 2010, 20, 4861. 